\title{
无配体参与的铱催化的苄位二级碳氢键的硼化反应
}

\author{
刘路华 $a, b \quad$ 杜荣荣 $a, b \quad$ 徐森苗*,a \\ ( ${ }^{a}$ 中国科学院兰州化学物理研究所 羰基合成与选择氧化国家重点实验室 兰州 730000) \\ ( ${ }^{b}$ 中国科学院大学 北京 100049)
}

\begin{abstract}
摘要 报道了无外加配体参与的以吡唑作为导向基团的铱催化的 $\mathrm{sp}^{3}$ 碳氢键的区域选择性嗍化反应. 在催化量的 $[\mathrm{Ir}(\mathrm{OMe})(\mathrm{cod})]_{2}$ 存在下，该反应能够顺利地将芐位的二级碳氢键转化成碳喼键. 该反应具有非常广谱的官能团兼容性, 能够以良好到优秀的产率生成相应的产物. 此外, 导向基团吡唑能够通过臭氧接转化成酰胺.

关键词碳氢键活化; 铱; 合成方法; 嗍化
\end{abstract}

\section{Ligand-Free Iridium-Catalyzed Borylation of Secondary Benzylic C-H Bonds}

\author{
Liu, Luhua ${ }^{a, b} \quad \mathrm{Du}$, Rongrong ${ }^{a, b} \quad \mathrm{Xu}$, Senmiao $^{*, a}$ \\ ( ${ }^{a}$ State Key Laboratory for Oxo Synthesis and Selective Oxidation, Lanzhou Institute of Chemical Physics, \\ Chinese Academy of Sciences, Lanzhou 730000) \\ ( ${ }^{b}$ University of Chinese Academy of Sciences, Beijing 100049)
}

\begin{abstract}
The ligand-free regioselective iridium-catalyzed $\mathrm{C}\left(\mathrm{sp}^{3}\right)-\mathrm{H}$ bond borylation using pyrazole as the directing group is reported. The reaction occurs smoothly at the secondary benzylic position in the presence of a catalytic amount of commercially available $[\operatorname{Ir}(\mathrm{OMe})(\mathrm{cod})]_{2}$. A variety of functionalities could be well tolerated, affording corresponding products in good to excellent yields. The pyrazole could be degraded into amide by ozonolysis.

Keywords $\mathrm{C}-\mathrm{H}$ activation; iridium; synthetic methods; borylation
\end{abstract}

\section{Introduction}

Ir- and Rh-catalyzed $\mathrm{C}-\mathrm{H}$ borylation has become a powerful tool in accessing enormous organoboronates via an atom- and step-economical approach. ${ }^{[1]}$ The regioselectivity is often governed by steric factors, occurring at sterically less congested positions. ${ }^{[2]}$ To achieve complementary regioselectivities, many strategies ${ }^{[3]}$ have been implemented through catalyst control, ${ }^{[4]}$ substrate control, ${ }^{[5]}$ hydrogen bond, ${ }^{[6]}$ and other means, ${ }^{[7-9]}$ which are particularly successful in reactions of aromatic substrates. But so far, regioselective $\mathrm{C}\left(\mathrm{sp}^{3}\right)-\mathrm{H}$ borylation still represents a formidable challenge. ${ }^{[3 \mathrm{~b}, 9-10]}$ For example, the $\mathrm{C}-\mathrm{H}$ borylation usually happens at the primary benzylic position, ${ }^{[\mathrm{c}]}$ while the borylation of secondary benzylic $\mathrm{C}-\mathrm{H}$ bonds remains underdeveloped. ${ }^{[11]}$ In this context, Hartwig and coworkers $^{[11]}$ developed a silyl directed secondary benzylic $\mathrm{C}-\mathrm{H}$ bond borylation. Key to the success of the reaction relies on the reversible formation of $\mathrm{Si}-\mathrm{Ir}$ covalent bond (Scheme 1a). The use of coordinating covalent bond for such transformation, however, remains unexplored.

Pyrazole has been recognized as an efficient directing group in $\mathrm{C}-\mathrm{H}$ functionalization, ${ }^{[12]}$ particularly in $\mathrm{Co}-{ }^{[13]}$

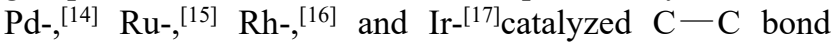
forming reactions. In stark contrast, its use in $\mathrm{C}$-heteroatom bond formation remains underdeveloped. ${ }^{[18]} \mathrm{We}$ herein disclose the first example of ligand-free Ir-catalyzed $\mathrm{C}-\mathrm{H}$ borylation at the secondary benzylic position using pyrazole as the directing group (Scheme 1b). We also demonstrate that the pyrazole group could be easily degraded into amide functionality.

\section{Results and discussion}

Our reaction commenced with the optimization of the reaction conditions of the pilot substrate 3,5-dimethyl-1phenethyl-1H-pyrazole (1). The reaction of $\mathbf{1}$ with 1.5

\footnotetext{
* Corresponding author. E-mail: senmiaoxu@licp.cas.cn

Received January 5, 2021; revised January 25, 2021; published online January 28, 2021.

Project supported by the National Natural Science Foundation of China (No. 21873261).

国家自然科学基金(No. 21873261)资助项目.
} 
a) Si-Ir covalent bond directed $\mathrm{C}\left(\mathrm{sp}^{3}\right)-\mathrm{H}$ borylation of secondary benzylic position

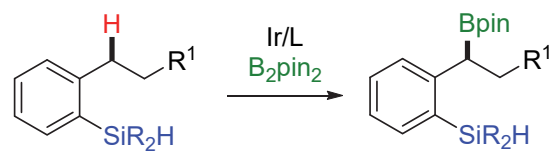

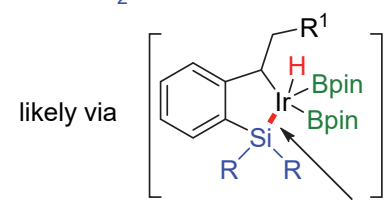

Si-Ir covalent bond

b) This work: N-Ir coordinate covalent bond directed $\mathrm{C}\left(\mathrm{sp}^{3}\right)-\mathrm{H}$ borylation of secondary benzylic position

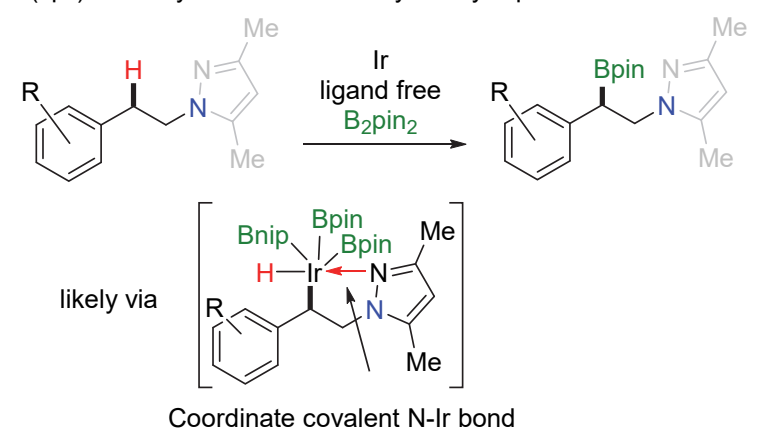

Scheme 1 Secondary benzylic $\mathrm{C}-\mathrm{H}$ bond borylation

equiv. of bis(pinacolato)diboron $\left(\mathrm{B}_{2} \mathrm{pin}_{2}\right)$ in the presence of $1 \mathrm{~mol} \% \quad[\operatorname{Ir}(\mathrm{OMe})(\mathrm{cod})]_{2}$ (cod: 1,5-cyclooctadiene) in $n$-heptane at $100{ }^{\circ} \mathrm{C}$ for $24 \mathrm{~h}$ resulted in complete conversion. Due to the instability of the borylated product 2 upon isolation by chromatography on silica gel, the crude reaction mixture was treated with $\mathrm{NaBO}_{3} \cdot 4 \mathrm{H}_{2} \mathrm{O}$ to afford 3 in $90 \%$ isolated yield (Table 1, Entry 1). Replacement of $[\operatorname{IrOMe}(\operatorname{cod})]_{2}$ with $[\mathrm{IrCl}(\mathrm{cod})]_{2}$ decreased the reactivity under otherwise identical reaction conditions (Table 1, Entry 2). In stark contrast, the use of pinacolborane (HBpin) gave only a trace amount of corresponding product (Table 1, Entry 3). Decreasing the $\mathrm{B}_{2} \mathrm{pin}_{2}$ loading to 1.0 equiv. resulted in a diminished yield of 3 (Table 1, Entry 4). The effect of the external ligand on the performance of the current reaction was also surveyed. For example, the use of 4,4'-di-tert-butyl-2,2'-bipyridine (dtbpy) furnished a mixture of phenyl ring borylated products rather than 3 (Table 1, Entry 5). The electron-deficient ligand $\mathrm{P}\left(\mathrm{C}_{6} \mathrm{~F}_{5}\right)_{3}$ did give the desired product, however, in inferior yield $(80 \%)$ compared to standard reaction conditions (Table 1 , Entry 6 vs Entry 1). When the reaction was carried out at $80{ }^{\circ} \mathrm{C}, 3$ was obtained in $60 \%$ yield (Table 1, Entry 7). The solvent effect revealed that $n$-heptane was optimal in terms of reactivity (Table 1, Entry 1 vs Entries 8 and 9). The diminished yield in $n$-hexane is probably caused by that the internal temperature could not reach $100{ }^{\circ} \mathrm{C}$ due to its lower boiling point.

With optimized reaction conditions in hand, the additional substrate scope of the current reaction as shown in Table 2 was then determined. Generally, most of the monosubstituted substrates could undergo the reaction
Table 1 Optimization of reaction conditions

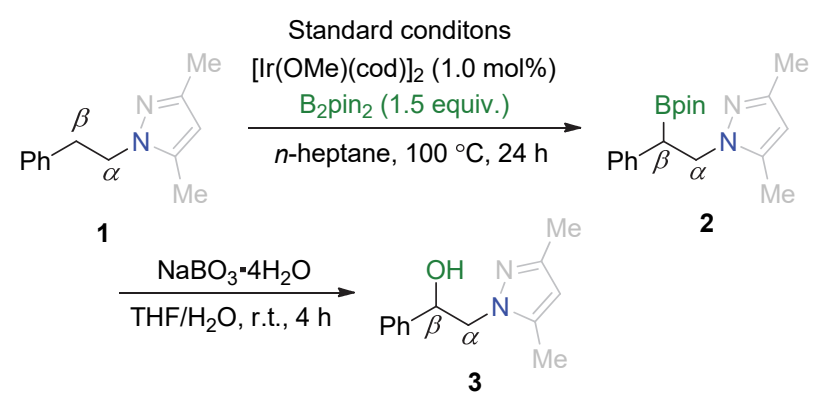

\begin{tabular}{|c|c|c|}
\hline Entry $^{a}$ & Variation from standard conditions & Yield $/ \%$ of $\mathbf{3}$ \\
\hline 1 & None & 90 \\
\hline 2 & {$[\mathrm{IrCl}(\operatorname{cod})]_{2}$ instead of $[\operatorname{IrOMe}(\operatorname{cod})]_{2}$} & 81 \\
\hline 3 & HBpin instead of $B_{2}$ pin 2 & Trace \\
\hline 4 & 1.0 equiv of $\mathrm{B}_{2}$ pin $_{2}$ was used & 50 \\
\hline 5 & 2 mol\% dtbpy was used & n.d. \\
\hline 6 & $2 \mathrm{~mol} \% \mathrm{P}\left(\mathrm{C}_{6} \mathrm{~F}_{5}\right)_{3}$ was used & 80 \\
\hline 7 & $80{ }^{\circ} \mathrm{C}$ instead of $100{ }^{\circ} \mathrm{C}$ & 60 \\
\hline 8 & 1,4-Dioxane instead of $n$-heptane & 33 \\
\hline 9 & $n$-Hexane instead of $n$-heptane & 77 \\
\hline
\end{tabular}

smoothly, giving the corresponding products $(\mathbf{3} \sim \mathbf{2 1})$ in good yields. Gratifyingly, sensitive functionalities such as ester and free hydroxy groups could also be well-tolerated, furnishing respective products $\mathbf{1 3}$ and $\mathbf{1 4}$ in $62 \%$ and $51 \%$ yields. The bond connections of compound $\mathbf{1 3}$ were unambiguously confirmed by single-crystal X-ray diffraction analysis. ${ }^{[19]}$ In addition, the reaction of substrates with a disubstituted phenyl group also occurred efficiently, providing corresponding products $\mathbf{2 2} \sim \mathbf{2 8}$ in $56 \% \sim 94 \%$ isolated yields. Finally, it was found that substrates bearing fused ring such as naphthyl and indolyl also showed good performance, giving corresponding products $29 \sim 31$ in $70 \% \sim 80 \%$ yields. Other substrates were also tested (Scheme 2). For example, the use of 1-phenethyl-1 $\mathrm{H}$-pyrazole (32) resulting in multiple mono- and di-borylated products; pyrazole derivatives $\mathbf{3 3} \sim \mathbf{3 5}$, however, showed very low or no reactivity. Substrates $\mathbf{3 6}$ and $\mathbf{3 7}$ did not give desired products under current reaction conditions.

To extend the synthetic applications of the current reaction, several transformations of crude borylated product 2 were conducted as shown in Scheme 3. For example, the pinacol group in $\mathbf{2}$ could be easily removed by treatment with $\mathrm{KHF}_{2}$ to provide difluroborane $\mathbf{3 8}$ in $70 \%$ yield. ${ }^{[20]}$ Fluorination of 2 with Selectfluor in the presence of a catalytic amount of $\mathrm{AgNO}_{3}$ rendered 39 in $77 \%$ yield. ${ }^{[21]}$ Homologation of 2 with $\mathrm{ICH}_{2} \mathrm{Cl} / n$-BuLi followed by oxidation afforded $\mathbf{4 0}$ in $70 \%$ yield. ${ }^{[22]}$ In addition, this crude borylated product could also undergo arylation to afford $\mathbf{4 1}$ in $61 \%$ yield. ${ }^{[23]}$ Importantly, pyrazole group in $\mathbf{3 7}$ could be degraded by ozonolysis to give acetamide $\mathbf{4 2}$ in $40 \%$ yield, ${ }^{[14 a]}$ which indicates that the advantage of pyrazole 
Table 2 Substrate scope ${ }^{a}$

3: $90 \%$ yield<smiles></smiles>

$$
\text { 8: } 80 \% \text { yield } \quad \text { 9: } 82 \% \text { yield } \quad 10: 53 \% \text { yield }
$$

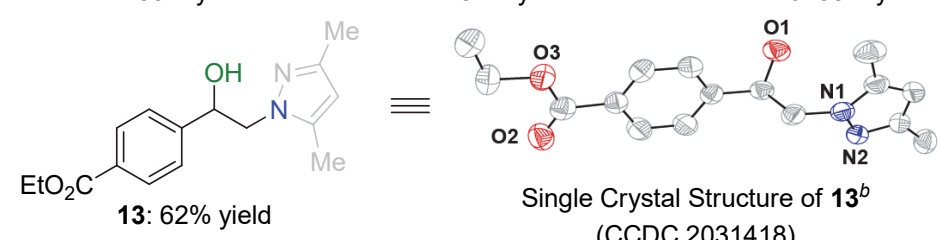
(CCDC 2031418)<smiles>Cc1cccc(C(O)Cn2nc([N+](=O)[O-])cc2C)c1</smiles>

16: $75 \%$ yield<smiles>Cc1cc([N+](=O)[O-])n(CC(O)c2cccc(C(F)(F)F)c2)n1</smiles>

21: $79 \%$ yield<smiles>COc1cccc(C(O)Cn2nc([N+](=O)[O-])cc2[N+](=O)[O-])c1</smiles>

17: $72 \%$ yield<smiles>Cc1cc(C)n(CC(O)c2ccc(C)c(C)c2)n1</smiles>

22: $81 \%$ yield<smiles>Cc1cc(C)n(CC(O)c2cccc(F)c2)n1</smiles>

18: $89 \%$ yield<smiles>Cc1cc(C)n(CC(O)c2ccc(F)c(F)c2)n1</smiles>

23: $94 \%$ yield<smiles>[Y9]c1cc([N+](=O)[O-])n(CC(O)c2cc(Cl)cc(Cl)c2)n1</smiles><smiles>Cc1cc(C)n(CC(O)c2ccc(OC(F)(F)F)cc2)n1</smiles>

11: $84 \%$ yield<smiles>[Y19]c1cc(C)n(CC(O)c2ccc(O)cc2)n1</smiles>

14: $51 \%$ yield<smiles>Cc1cc([N+](=O)[O-])n(CC(O)c2ccc(-c3ccccc3)cc2)n1</smiles>

12: $61 \%$ yield<smiles>CCCCOc1ccc(C(O)Cn2nc(C)cc2C)cc1</smiles>

15: $86 \%$ yield<smiles>[N+]=[N+]([O-])c1cc([N+](=O)[O-])nn1CC(O)c1cccc(Cl)c1</smiles>

19: $90 \%$ yield<smiles>Cc1cc(C)n(CC(O)c2ccc(Cl)c(Cl)c2)n1</smiles>

24: $83 \%$ yield<smiles>Cc1cc(C)n(CC(O)c2ccc3ccccc3c2)n1</smiles>

29: $70 \%$ yield

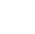<smiles>Cc1cc([N+](=O)[O-])n(CC(O)c2cccc(OC(F)(F)F)c2)n1</smiles>

20: $71 \%$ yield<smiles>Cc1cc([N+](=O)[O-])n(CC(O)c2ccc3c(c2)CCO3)n1</smiles>

25: $82 \%$ yield<smiles>Cc1cc(C)n(CC(O)c2cccc3ccccc23)n1</smiles>

30: $80 \%$ yield<smiles>Cc1cc(C)n(CC(O)c2cn(C)c3ccccc23)n1</smiles>

27: $63 \%$ yield

28: $56 \%$ yield 
<smiles>c1ccccc1</smiles>

32

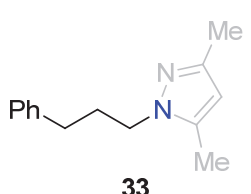

33

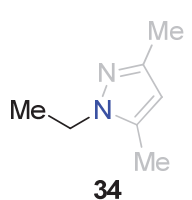

34

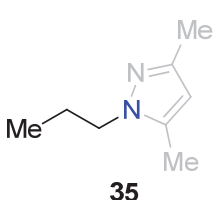

35

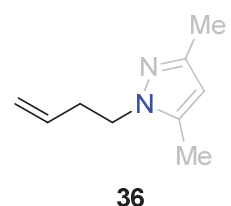

36

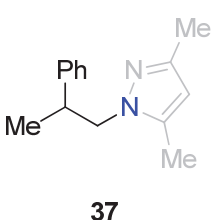

37

Scheme 2 Substrates do not work well

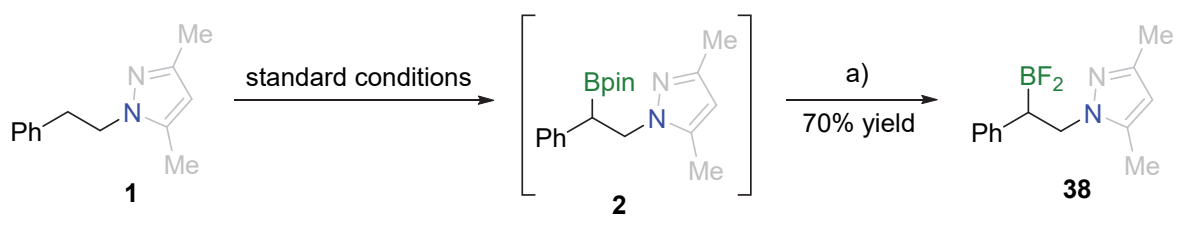

b) $77 \%$ yield<smiles>CC(=O)NCC(F)c1ccccc1</smiles>

42 c) $70 \%$ yield

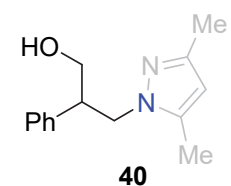

$61 \%$ yield d)

Reagents and conditions: a) $\mathrm{KHF}_{2}, \mathrm{MeOH} / \mathrm{H}_{2} \mathrm{O}$, r.t., 2 h. b) $\mathrm{AgNO}_{3}(0.2 \mathrm{~mol} \%$ ), Selectfluor TFA, THF, $60{ }^{\circ} \mathrm{C}, 6$ h. c) $I \mathrm{ICH}_{2} \mathrm{Cl} / n-\mathrm{BuLi},-78^{\circ} \mathrm{C}, 10 \mathrm{~min}$, then $\mathrm{NaBO}_{3} 4 \mathrm{H}_{2} \mathrm{O}$. THF/ $\mathrm{H}_{2} \mathrm{O}$, r.t., 4 h. d) thiophene, $n$-BuLi, THF, $-78{ }^{\circ} \mathrm{C}, 2$ h, then NBS, $-78{ }^{\circ} \mathrm{C} \sim$ r.t., 2.5 h. e) $\mathrm{O}_{3}$, pyridine, MeOAc, $-78^{\circ} \mathrm{C}, 1 \mathrm{~h}$, then $\mathrm{NiCl}_{2}, \mathrm{NaBH}_{4}, \mathrm{MeOH},-78^{\circ} \mathrm{C} \sim$ r.t., 19 h.

Scheme 3 Transformation of crude borylated product 2

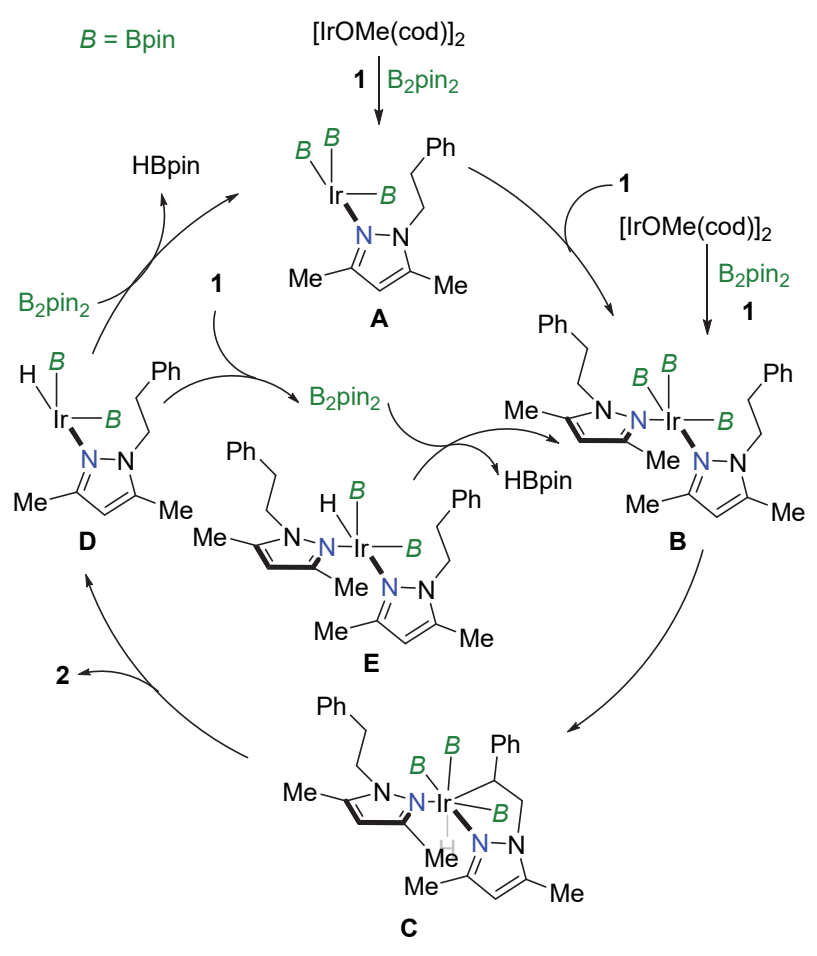

Figure 1 Plausible reaction mechanism

$\operatorname{Ir}(\mathrm{V})$ complex C. Reductive elimination of $\mathbf{C}$ could generate the borylated product $\mathbf{2}$ along with the formation of hydrido boryl Ir(III) complex D. Reaction of $\mathbf{D}$ with $\mathrm{B}_{2} \mathrm{pin}_{2}$ could regenerate complex A. Alternatively, complex $\mathbf{B}$ could also be formed initially by the reaction of $[\operatorname{Ir}(\mathrm{OMe})(\mathrm{cod})]_{2}$ with $\mathrm{B}_{2} \mathrm{pin}_{2}$ and two molecules of $\mathbf{1}$ and then undergo similar steps to furnish 2. Another plausible scenario is that complex $\mathbf{D}$ could be coordinated with $\mathbf{1}$ to form intermediate $\mathbf{E}$. Reaction of $\mathbf{E}$ with $\mathrm{B}_{2} \mathrm{pin}_{2}$ to regenerate complex $\mathbf{B}$ for the next catalytic cycle. The formation of HBpin was evidenced by ${ }^{11} \mathrm{~B}$ NMR of the crude reaction mixture (See the Supporting Information for more details).

\section{Conclusions}

In summary, we have developed the first example of ligand-free Ir-catalyzed borylation of seondary benzylic $\mathrm{C}-\mathrm{H}$ bonds using pyrazole as the directing group. A variety of functional groups could be well-tolerated, affording benzylic functionalized products in good yields. We also demonstrated that the crude borylated product could undergo a variety of transformations, including $\mathrm{C}$ $\mathrm{F}$ and $\mathrm{C}-\mathrm{C}$ bond forming reactions. Importantly, the pyrazole group could be readily converted to amide functionality, indicating the potential of the current method in the synthesis of 1,2-functionalized amine derivatives. Further application of the obtained products and the development of the other context of ligand-free $\mathrm{C}-\mathrm{H}$ functionalization reactions are currently underway in our laboratory.

\section{Experimental section}

Pyrazole substrates were prepared according to the liter- 
ature procedures. ${ }^{[24]} \mathrm{B}_{2}$ pin $_{2}$ was recrystallization from $n$-hexane prior to use. All other chemicals and solvents were used as received. ${ }^{1} \mathrm{H} \mathrm{NMR},{ }^{13} \mathrm{C}$ NMR, ${ }^{19} \mathrm{~F}$ NMR and ${ }^{11} \mathrm{~B}$ NMR spectra were recorded on a Zhongke-Niujin 400, and a Bruker DRX400 spectrometers at ambient temperature with $\mathrm{CDCl}_{3}$ as solvent. ${ }^{13} \mathrm{C}$ shifts were obtained with ${ }^{1} \mathrm{H}$ decoupling. High-resolution mass spectroscopies (HRMS) were obtained on Agilent 6530, Agilent 6224 TOF LC/MS, and Agilent 7205 GCQTOF spectrometers. $\mathrm{X}$-ray crystallography was measured on an XtaLAB AFC12 (RINC): Kappa dual home/near diffractometer. Melting points were determined on an Electrothermal IA9000 Series Digital Melting Point Apparatus.

4.1 General procedure for borylation of secondary benzylic $\mathrm{C}-\mathrm{H}$ bonds

To a $25 \mathrm{~mL}$ flame-dried Schlenk tube in $\mathrm{N}_{2}$-filled glove-box charged with pyrazole substrate $(0.2 \mathrm{mmol})$ and $[\operatorname{Ir}(\mathrm{OMe})(\mathrm{cod})]_{2} \quad(1.3 \mathrm{mg}, \quad 0.002 \mathrm{mmol})$ was added $n$-heptane $(2.0 \mathrm{~mL})$. The resulting mixture was allowed to stir at $100{ }^{\circ} \mathrm{C}$ for $24 \mathrm{~h}$. After cooled to room temperature and concentrated, the residue was treated with $\mathrm{NaBO}_{3}$. $4 \mathrm{H}_{2} \mathrm{O}(0.15 \mathrm{~g}, 1.0 \mathrm{mmol})$ in $\mathrm{THF} / \mathrm{H}_{2} \mathrm{O}(V: V=1: 1,4$ $\mathrm{mL}$ ) for $4 \mathrm{~h}$ at room temperature. The mixture were extracted with EtOAc $(10 \mathrm{~mL} \times 3)$. The combined organic phase was then dried over anhydrous $\mathrm{Na}_{2} \mathrm{SO}_{4}$. After removal of the solvent, the residue was purified by column chromatography on silica gel using petroleum ether/ EtOAc as the eluent to afford 2-pyrazol-1-yl-1-arylethanol.

2-(3,5-Dimethyl-1H-pyrazol-1-yl)-1-phenylethanol (3): $R_{\mathrm{f}}=0.2[V($ petroleum ether $): V($ EtOAc $)=4: 1], 38.9 \mathrm{mg}$, $90 \%$ yield. White solid, m.p. $143.7 \sim 146.2{ }^{\circ} \mathrm{C}$; ${ }^{1} \mathrm{H}$ NMR $\left(400 \mathrm{MHz}, \mathrm{CDCl}_{3}\right) \delta: 7.36 \sim 7.29(\mathrm{~m}, 5 \mathrm{H}), 5.78(\mathrm{~s}, 1 \mathrm{H})$, 5.08 (dd, $J=8.0,3.2 \mathrm{~Hz}, 1 \mathrm{H}), 4.80$ (brs, $1 \mathrm{H}), 4.14$ (dd, $J=$ 13.6, $2.8 \mathrm{~Hz}, 1 \mathrm{H}), 4.02$ (dd, $J=14.0,8.0 \mathrm{~Hz}, 1 \mathrm{H}), 2.24$ (s, $3 \mathrm{H}), 2.01(\mathrm{~s}, 3 \mathrm{H}) ;{ }^{13} \mathrm{C} \mathrm{NMR}\left(100 \mathrm{MHz}, \mathrm{CDCl}_{3}\right) \delta: 148.2$, $141.1,139.9,128.5,127.97,125.9$ 105.0, 73.5, 54.9, 13.4, 10.7; HRMS (ESI-TOF) calcd for $\mathrm{C}_{13} \mathrm{H}_{16} \mathrm{~N}_{2} \mathrm{NaO}[\mathrm{M}+$ $\mathrm{Na}]^{+}$239.1155, found 239.1164.

2-(3,5-Dimethyl-1H-pyrazol-1-yl)-1-(o-tolyl)ethanol (4): $R_{\mathrm{f}}=0.2[V($ petroleum ether $): V($ EtOAc $)=4: 1], 34.5 \mathrm{mg}$, $75 \%$ yield. White solid, m.p. $123.2 \sim 125.9{ }^{\circ} \mathrm{C} ;{ }^{1} \mathrm{H}$ NMR $\left(400 \mathrm{MHz}, \mathrm{CDCl}_{3}\right) \delta: 7.46 \sim 7.44(\mathrm{~m}, 1 \mathrm{H}), 7.24 \sim 7.13(\mathrm{~m}$, $3 \mathrm{H}), 5.80(\mathrm{~s}, 1 \mathrm{H}), 5.28(\mathrm{dd}, J=8.0,2.8 \mathrm{~Hz}, 1 \mathrm{H}), 4.77$ (brs, $1 \mathrm{H}), 4.12$ (dd, $J=14.0,2.8 \mathrm{~Hz}, 1 \mathrm{H}), 3.98$ (dd, $J=14.0,8.0$ $\mathrm{Hz}, 1 \mathrm{H}), 2.35$ (s, 3H), 2.25 (s, 3H), $2.02(\mathrm{~s}, 3 \mathrm{H}) ;{ }^{13} \mathrm{C}$ NMR $\left(100 \mathrm{MHz}, \mathrm{CDCl}_{3}\right) \delta: 148.3,139.8,139.0,134.2,130.3$, 127.6, 126.4, 125.9, 105.1, 70.1, 53.5, 19.0, 13.4, 10.7; HRMS (ESI-TOF) calcd for $\mathrm{C}_{14} \mathrm{H}_{18} \mathrm{~N}_{2} \mathrm{NaO}[\mathrm{M}+\mathrm{Na}]^{+}$ 253.1311, found 253.1318.

2-(3,5-Dimethyl-1H-pyrazol-1-yl)-1-( $p$-tolyl)ethanol (5): $R_{\mathrm{f}}=0.2[V($ petroleum ether $): V($ EtOAc $)=4: 1], 34.5 \mathrm{mg}$, $75 \%$ yield. White solid, m.p. $151.0 \sim 151.5{ }^{\circ} \mathrm{C} ;{ }^{1} \mathrm{H}$ NMR $\left(400 \mathrm{MHz}, \mathrm{CDCl}_{3}\right) \delta: 7.21(\mathrm{~d}, J=8.0 \mathrm{~Hz}, 2 \mathrm{H}), 7.15(\mathrm{~d}, J=$ $7.6 \mathrm{~Hz}, 2 \mathrm{H}), 5.79(\mathrm{~s}, 1 \mathrm{H}), 5.05$ (dd, $J=8.0,2.0 \mathrm{~Hz}, 1 \mathrm{H})$, 4.67 (brs, 1H), 4.12 (dd, $J=14.0,3.2 \mathrm{~Hz}, 1 \mathrm{H}), 4.00$ (dd, $J=14.0,8.0 \mathrm{~Hz}, 1 \mathrm{H}), 2.34$ (s, 3H), 2.24 (s, 3H), 2.03 (s,
$3 \mathrm{H}) ;{ }^{13} \mathrm{C} \mathrm{NMR}\left(100 \mathrm{MHz}, \mathrm{CDCl}_{3}\right) \delta: 148.1,139.9,138.1$, 137.6, 129.1, 125.8, 105.0, 73.3, 55.0, 21.1, 13.4, 10.8; HRMS (ESI-TOF) calcd for $\mathrm{C}_{14} \mathrm{H}_{19} \mathrm{~N}_{2} \mathrm{O}[\mathrm{M}+\mathrm{H}]{ }^{+}$ 231.1492, found 231.1502.

2-(3,5-Dimethyl-1H-pyrazol-1-yl)-1-(4-methoxyphenyl)ethanol (6): $R_{\mathrm{f}}=0.2[V($ petroleum ether $): V($ EtOAc $)=$ $4: 1], 44.3 \mathrm{mg}, 90 \%$ yield. White solid, m.p. 181.2 $184.7{ }^{\circ} \mathrm{C} ;{ }^{1} \mathrm{H}$ NMR (400 MHz, $\left.\mathrm{CDCl}_{3}\right) \delta: 7.24$ (d, $J=8.4$ $\mathrm{Hz}, 2 \mathrm{H}), 6.88$ (d, J=8.4 Hz, 2H), 5.79 (s, 1H), 5.03 (dd, $J=8.0,2.8 \mathrm{~Hz}, 1 \mathrm{H}), 4.64$ (brs, $1 \mathrm{H}), 4.11$ (dd, $J=14.0,3.2$ $\mathrm{Hz}, 1 \mathrm{H}), 4.00$ (dd, $J=14.0,8.0 \mathrm{~Hz}, 1 \mathrm{H}), 3.80$ (s, 3H), 2.24 $(\mathrm{s}, 3 \mathrm{H}), 2.04(\mathrm{~s}, 3 \mathrm{H}) ;{ }^{13} \mathrm{C} \mathrm{NMR}\left(100 \mathrm{MHz}, \mathrm{CDCl}_{3}\right) \delta$ : 159.3, 148.2, 139.9, 133.3, 127.1, 113.9, 105.0, 73.1, 55.3, 55.0, 13.4, 10.8; HRMS (ESI-TOF) calcd for $\mathrm{C}_{14} \mathrm{H}_{18} \mathrm{~N}_{2-}$ $\mathrm{NaO}_{2}[\mathrm{M}+\mathrm{Na}]^{+}$269.1260, found 269.1256.

2-(3,5-Dimethyl-1H-pyrazol-1-yl)-1-(4-fluorophenyl)ethanol (7): $R_{\mathrm{f}}=0.2[V($ petroleum ether $): V($ EtOAc $)=4$ : 1], $35.1 \mathrm{mg}, 75 \%$ yield. White solid, m.p. 140.1 $141.2{ }^{\circ} \mathrm{C}$; ${ }^{1} \mathrm{H}$ NMR (400 MHz, $\left.\mathrm{CDCl}_{3}\right) \delta: 7.29 \sim 7.25(\mathrm{~m}$, $2 \mathrm{H}), 7.04 \sim 7.00(\mathrm{~m}, 2 \mathrm{H}), 5.78(\mathrm{~s}, 1 \mathrm{H}), 5.07$ (dd, $J=7.6$, $2.8 \mathrm{~Hz}, 1 \mathrm{H}), 4.90$ (brs, 1H), 4.12 (dd, $J=14.0,2.8 \mathrm{~Hz}$, $1 \mathrm{H}), 3.98$ (dd, $J=14.0,8.0 \mathrm{~Hz}, 1 \mathrm{H}), 2.24(\mathrm{~s}, 3 \mathrm{H}), 2.01$ (s, $3 \mathrm{H}) ;{ }^{13} \mathrm{C} \mathrm{NMR}\left(100 \mathrm{MHz}, \mathrm{CDCl}_{3}\right) \delta: 162.4(\mathrm{~d}, J=244.0)$, 148.3, 139.9, 136.9 (d, $J=4.6 \mathrm{~Hz}), 127.6$ (d, $J=8.1 \mathrm{~Hz})$, $115.3(\mathrm{~d}, J=21.4 \mathrm{~Hz}), 105.1,72.9,54.7,13.4,10.7 ;{ }^{19} \mathrm{~F}$ NMR $\left(376 \mathrm{MHz}, \mathrm{CDCl}_{3}\right) \delta$ : -114.6 ; HRMS (ESI-TOF) calcd for $\mathrm{C}_{13} \mathrm{H}_{15} \mathrm{FN}_{2} \mathrm{NaO}[\mathrm{M}+\mathrm{Na}]^{+}$257.1061, found 257.1055 .

1-(4-Chlorophenyl)-2-(3,5-dimethyl-1H-pyrazol-1-yl)ethanol (8): $R_{\mathrm{f}}=0.2[V($ petroleum ether $): V($ EtOAc $)=4$ : 1], $40.1 \mathrm{mg}, 80 \%$ yield. White solid, m.p. $138.4 \sim$ $141.2{ }^{\circ} \mathrm{C} ;{ }^{1} \mathrm{H}$ NMR (400 MHz, $\left.\mathrm{CDCl}_{3}\right) \delta: 7.31$ (d, $J=8.4$ $\mathrm{Hz}, 2 \mathrm{H}), 7.23$ (d, $J=8.4 \mathrm{~Hz}, 2 \mathrm{H}), 5.78(\mathrm{~s}, 1 \mathrm{H}), 5.06$ (dd, $J=7.6,2.8 \mathrm{~Hz}, 1 \mathrm{H}), 4.98$ (br, 1H), 4.12 (dd, $J=14.0,2.8$ $\mathrm{Hz}, 1 \mathrm{H}), 3.98$ (dd, $J=14.0,7.6 \mathrm{~Hz}, 1 \mathrm{H}), 2.23$ (s, 3H), 2.01 $(\mathrm{s}, 3 \mathrm{H}) ;{ }^{13} \mathrm{C} \mathrm{NMR}\left(100 \mathrm{MHz}, \mathrm{CDCl}_{3}\right) \delta: 148.3,139.9$, 139.7, 133.6, 128.0, 127.3, 105.1, 72.8, 54.6, 13.4, 10.7; HRMS (ESI-TOF) calcd for $\mathrm{C}_{13} \mathrm{H}_{16} \mathrm{ClN}_{2} \mathrm{O}[\mathrm{M}+\mathrm{H}]^{+}$ 251.0946, found 251.0944 .

1-(4-Bromophenyl)-2-(3,5-dimethyl-1 $H$-pyrazol-1-yl)ethanol (9): $R_{\mathrm{f}}=0.2$ [ $V$ (petroleum ether) $: V($ EtOAc $)=4$ : 1], $48.4 \mathrm{mg}, 82 \%$ yield. Yellow solid, m.p. 146.5 $148.6{ }^{\circ} \mathrm{C}$; ${ }^{1} \mathrm{H}$ NMR (400 MHz, $\left.\mathrm{CDCl}_{3}\right) \delta: 7.46$ (d, $J=8.4$ $\mathrm{Hz}, 2 \mathrm{H}), 7.17$ (d, $J=8.4 \mathrm{~Hz}, 2 \mathrm{H}), 5.78$ (s, 1H), 5.04 (dd, $J=7.6,2.8 \mathrm{~Hz}, 1 \mathrm{H}), 5.00$ (brs, 1H) 4.12 (dd, $J=14.0,3.2$ $\mathrm{Hz}, 1 \mathrm{H}), 3.97$ (dd, $J=14.0,7.6 \mathrm{~Hz}, 1 \mathrm{H}), 2.23$ (s, 3H), 2.00 $(\mathrm{s}, 3 \mathrm{H}) ;{ }^{13} \mathrm{C}$ NMR $\left(100 \mathrm{MHz}, \mathrm{CDCl}_{3}\right) \delta: 148.3,140.2$, 139.9, 131.5, 127.6, 121.7, 105.1, 72.9, 54.5, 13.4, 10.7; HRMS (ESI-TOF) calcd for $\mathrm{C}_{13} \mathrm{H}_{15} \mathrm{BrN}_{2} \mathrm{NaO}[\mathrm{M}+\mathrm{Na}]^{+}$ 317.0260, found 317.0270.

2-(3,5-Dimethyl-1H-pyrazol-1-yl)-1-(4-(trifluoromethyl)phenyl)ethanol (10): $R_{\mathrm{f}}=0.2 \quad[V$ (petroleum ether) : $V($ EtOAc $)=4: 1]$, $30.1 \mathrm{mg}, 53 \%$ yield. Colorless solid, m.p. $155.2 \sim 157.0{ }^{\circ} \mathrm{C} ;{ }^{1} \mathrm{H}$ NMR $\left(400 \mathrm{MHz}, \mathrm{CDCl}_{3}\right) \delta$ : $7.60(\mathrm{~d}, J=8.0 \mathrm{~Hz}, 2 \mathrm{H}), 7.43$ (d, $J=8.4 \mathrm{~Hz}, 2 \mathrm{H}), 5.79$ (s, $1 \mathrm{H}), 5.14(\mathrm{~d}, J=7.6 \mathrm{~Hz}, 2 \mathrm{H}), 4.17(\mathrm{dd}, J=14.0,2.8 \mathrm{~Hz}$, $1 \mathrm{H}), 4.00(\mathrm{dd}, J=14.0,7.6 \mathrm{~Hz}, 1 \mathrm{H}), 2.24$ (s, 3H), 2.00 (s, 
$3 \mathrm{H}) ;{ }^{13} \mathrm{C}$ NMR $\left(100 \mathrm{MHz}, \mathrm{CDCl}_{3}\right) \delta: 148.5,145.2,139.9$, 130.1 (q, $J=32.4 \mathrm{~Hz}), 126.3,125.4$ (q, $J=3.6 \mathrm{~Hz}), 124.1$ $(\mathrm{q}, J=272.4 \mathrm{~Hz}), 105.2,73.0,54.4,13.4,10.7 ;{ }^{19} \mathrm{~F}$ NMR $\left(376 \mathrm{MHz}, \mathrm{CDCl}_{3}\right) \delta$ : -62.5 ; HRMS (ESI-TOF) calcd for $\mathrm{C}_{14} \mathrm{H}_{16} \mathrm{~F}_{3} \mathrm{~N}_{2} \mathrm{O} \quad[\mathrm{M}+\mathrm{H}]^{+}$285.1209, found 285.1200.

2-(3,5-Dimethyl-1H-pyrazol-1-yl)-1-(4-(trifluoromethoxy)phenyl)ethanol (11): $R_{\mathrm{f}}=0.2[V$ (petroleum ether) : $V($ EtOAc $)=4: 1], 50.4 \mathrm{mg}, 84 \%$ yield. White solid, m.p. $141.1 \sim 142.4{ }^{\circ} \mathrm{C} ;{ }^{1} \mathrm{H}$ NMR $\left(400 \mathrm{MHz}, \mathrm{CDCl}_{3}\right) \delta: 7.33(\mathrm{~d}$, $J=8.8 \mathrm{~Hz}, 2 \mathrm{H}), 7.19$ (d, $J=8.0 \mathrm{~Hz}, 2 \mathrm{H}), 5.79$ (s, 1H), 5.10 (dd, $J=7.6,2.8 \mathrm{~Hz}, 1 \mathrm{H}), 5.03$ (brs, 1H), 4.13 (dd, $J=14.0$, $2.8 \mathrm{~Hz}, 1 \mathrm{H}), 3.99$ (dd, $J=14.0,7.6 \mathrm{~Hz}, 1 \mathrm{H}), 2.24$ (s, 3H), $2.00(\mathrm{~s}, 3 \mathrm{H}) ;{ }^{13} \mathrm{C} \mathrm{NMR}\left(100 \mathrm{MHz}, \mathrm{CDCl}_{3}\right) \delta: 148.8,148.4$, $139.9,139.8,127.4,121.0,120.5$ (d, $J=255.5 \mathrm{~Hz}) 105.1$, 72.8, 54.6, 13.4, 10.7; ${ }^{19} \mathrm{~F}$ NMR (377 $\left.\mathrm{MHz}, \mathrm{CDCl}_{3}\right) \delta:-$ 57.9; HRMS (ESI-TOF) calcd for $\mathrm{C}_{14} \mathrm{H}_{16} \mathrm{~F}_{3} \mathrm{~N}_{2} \mathrm{O}_{2}{ }^{+}[\mathrm{M}+\mathrm{H}]$ ${ }^{+} 301.1158$, found 301.1162 .

1-([1,1'-Biphenyl]-4-yl)-2-(3,5-dimethyl-1H-pyrazol-1yl)ethanol (12): $R_{\mathrm{f}}=0.2[V($ petroleum ether $): V($ EtOAc $)=$ $4: 1], 35.6 \mathrm{mg}, 61 \%$ yield. White solid, m.p. 169.9 $172.2{ }^{\circ} \mathrm{C} ;{ }^{1} \mathrm{H}$ NMR (400 MHz, $\left.\mathrm{CDCl}_{3}\right) \delta: 7.60 \sim 7.57(\mathrm{~m}$, $4 \mathrm{H}), 7.46 \sim 7.33(\mathrm{~m}, 5 \mathrm{H}), 5.80(\mathrm{~s}, 1 \mathrm{H}), 5.14(\mathrm{dd}, J=8.0$, $2.8 \mathrm{~Hz}, 1 \mathrm{H}), 4.84$ (s, 1H), 4.19 (dd, $J=14.0,3.2 \mathrm{~Hz}, 1 \mathrm{H})$, 4.06 (dd, $J=14.0,8.0 \mathrm{~Hz}, 1 \mathrm{H}), 2.26$ (s, 3H), 2.05 (s, 3H); ${ }^{13} \mathrm{C}$ NMR (100 MHz, $\left.\mathrm{CDCl}_{3}\right) \delta: 148.2,140.8,140.7,140.1$, 139.9, 128.8, 127.3, 127.2, 127.1, 126.4, 105.0, 73.3, 54.8, 13.4, 10.8; HRMS (ESI-TOF) calcd for $\mathrm{C}_{19} \mathrm{H}_{21} \mathrm{~N}_{2} \mathrm{O}[\mathrm{M}+$ $\mathrm{H}^{+}$293.1648, found 293.1647.

Ethyl 4-(2-(3,5-dimethyl-1H-pyrazol-1-yl)-1-hydroxyethyl)benzoate (13): $R_{\mathrm{f}}=0.15 \quad[V$ (petroleum ether) : $V($ EtOAc $)=4: 1], 35.7 \mathrm{mg}, 62 \%$ yield. White solid, m.p. $122.3 \sim 125.2{ }^{\circ} \mathrm{C} ;{ }^{1} \mathrm{H}$ NMR (400 MHz, $\left.\mathrm{CDCl}_{3}\right) \delta: 8.01(\mathrm{~d}$, $J=8.4 \mathrm{~Hz}, 2 \mathrm{H}), 7.36$ (d, $J=8.4 \mathrm{~Hz}, 2 \mathrm{H}), 5.77$ (s, 1H), 5.14 (dd, $J=7.2,2.8 \mathrm{~Hz}, 1 \mathrm{H}), 5.07(\mathrm{br}, 1 \mathrm{H}), 4.37$ (q, $J=7.2 \mathrm{~Hz}$, 2H), 4.18 (dd, $J=14.0,2.8 \mathrm{~Hz}, 1 \mathrm{H}), 4.02(\mathrm{dd}, J=14.0,7.2$ $\mathrm{Hz}, 1 \mathrm{H}), 2.24(\mathrm{~s}, 3 \mathrm{H}), 1.97(\mathrm{~s}, 3 \mathrm{H}), 1.39(\mathrm{t}, J=7.2 \mathrm{~Hz}$, $3 \mathrm{H}) ;{ }^{13} \mathrm{C}$ NMR $\left(100 \mathrm{MHz}, \mathrm{CDCl}_{3}\right) \delta: 166.3,148.3,146.3$, $140.0,129.9,129.6,125.8,105.0,73.0,60.9,54.4,14.3$, 13.3, 10.7; HRMS (ESI-TOF) calcd for $\mathrm{C}_{16} \mathrm{H}_{21} \mathrm{~N}_{2} \mathrm{O}_{3}[\mathrm{M}+$ $\mathrm{H}]^{+}$289.1547, found 289.1545 .

4-(2-(3,5-Dimethyl-1H-pyrazol-1-yl)-1-hydroxyethyl)phenol (14): $R_{\mathrm{f}}=0.2[V($ petroleum ether $): V($ EtOAc $)=$ $1: 1], 23.7 \mathrm{mg}, 51 \%$ yield. White solid, m.p. 158.6 $160.2{ }^{\circ} \mathrm{C} ;{ }^{1} \mathrm{H}$ NMR (400 MHz, $\left.\mathrm{CD}_{3} \mathrm{OD}\right) \delta: 7.07$ (d, $J=8.4$ $\mathrm{Hz}, 2 \mathrm{H}), 6.72$ (d, J=8.4 Hz, 2H), 5.74 (s, 1H), 4.92 (dd, $J=6.8,6.4 \mathrm{~Hz}, 1 \mathrm{H}), 4.57$ (brs, $1 \mathrm{H}), 4.16$ (dd, $J=14.0,7.6$ $\mathrm{Hz}, 1 \mathrm{H}), 3.96$ (dd, $J=14.0,6.0 \mathrm{~Hz}, 1 \mathrm{H}), 2.18$ (s, 3H), 1.94 $(\mathrm{s}, 3 \mathrm{H}) ;{ }^{13} \mathrm{C} \mathrm{NMR}\left(100 \mathrm{MHz}, \mathrm{CD}_{3} \mathrm{OD}\right) \delta: 158.2,148.8$, $142.3,134.2,128.3,116.1,105.7,74.1,56.6,13.2,10.8$; HRMS (ESI-TOF) calcd for $\mathrm{C}_{13} \mathrm{H}_{17} \mathrm{~N}_{2} \mathrm{O}_{2}[\mathrm{M}+\mathrm{H}]^{+}$ 233.1285, found 233.1285.

1-(4-((tert-Butyldimethylsilyl)oxy)phenyl)-2-(3,5-dimethyl-1H-pyrazol-1-yl)ethanol (15): $R_{\mathrm{f}}=0.2[V$ (petroleum ether) $: V($ EtOAc $)=4: 1], 59.6 \mathrm{mg}, 86 \%$ yield. Yellow oil. ${ }^{1} \mathrm{H}$ NMR $\left(400 \mathrm{MHz}, \mathrm{CDCl}_{3}\right) \delta: 7.14(\mathrm{~d}, J=8.4 \mathrm{~Hz}$, 2H), $6.80(\mathrm{~d}, J=8.8 \mathrm{~Hz}, 2 \mathrm{H}), 5.77(\mathrm{~s}, 1 \mathrm{H}), 5.01$ (dd, $J=$ 8.0, $3.8 \mathrm{~Hz}, 1 \mathrm{H}), 4.65$ (brs, $1 \mathrm{H}), 4.11$ (dd, $J=14.0,3.2 \mathrm{~Hz}$,
1H), 4.00 (dd, $J=14.0,7.6 \mathrm{~Hz}, 1 \mathrm{H}), 2.24$ (s, 3H), 2.00 (s, $3 \mathrm{H}), 0.98(\mathrm{~s}, 9 \mathrm{H}), 0.18(\mathrm{~s}, 6 \mathrm{H}) ;{ }^{13} \mathrm{C} \mathrm{NMR}(100 \mathrm{MHz}$, $\left.\mathrm{CDCl}_{3}\right) \delta: 155.4,148.1,139.9,133.9,127.0,120.1,104.9$, 73.1, 54.9, 25.7, 18.2, 13.4, 10.7, -4.5; HRMS (ESI-TOF) calcd for $\mathrm{C}_{19} \mathrm{H}_{31} \mathrm{~N}_{2} \mathrm{O}_{2} \mathrm{Si}[\mathrm{M}+\mathrm{H}]^{+}$347.2149, found 347.2152 .

2-(3,5-Dimethyl-1H-pyrazol-1-yl)-1-( $m$-tolyl)ethanol (16): $R_{\mathrm{f}}=0.2[V($ petroleum ether $): V($ EtOAc $)=4: 1]$, $34.5 \mathrm{mg}, 75 \%$ yield. White solid, m.p. 123.2 $124.7{ }^{\circ} \mathrm{C}$; ${ }^{1} \mathrm{H}$ NMR (400 MHz, $\left.\mathrm{CDCl}_{3}\right) \delta: 7.23(\mathrm{t}, J=7.6 \mathrm{~Hz}, 1 \mathrm{H})$, 7.15 (s, 1H), 7.14 (d, $J=7.6 \mathrm{~Hz}, 1 \mathrm{H}), 7.10$ (d, $J=6.4 \mathrm{~Hz}$, 1H), 5.79 (s, 1H), 5.04 (dd, $J=8.4,2.8 \mathrm{~Hz}, 1 \mathrm{H}), 4.69$ (brs, $1 \mathrm{H}), 4.13$ (dd, $J=14.0,2.8 \mathrm{~Hz}, 1 \mathrm{H}), 4.00$ (dd, $J=14.0,8.0$ $\mathrm{Hz}, 1 \mathrm{H}), 2.35$ (s, 3H), 2.25 (s, 3H), 2.05 (s, 3H); ${ }^{13} \mathrm{C}$ NMR $\left(100 \mathrm{MHz} \mathrm{CDCl}_{3}\right) \delta: 148.2,140.9,139.9,138.2,128.6$, 128.4, 126.6, 123.0, 105.0, 73.5, 54.9, 21.4, 13.4, 10.8; HRMS (ESI-TOF) calcd for $\mathrm{C}_{14} \mathrm{H}_{19} \mathrm{~N}_{2} \mathrm{O}[\mathrm{M}+\mathrm{H}]$ 231.1492, found 231.1496.

2-(3,5-Dimethyl-1H-pyrazol-1-yl)-1-(3-methoxyphenyl)ethanol (17): $R_{\mathrm{f}}=0.2[V($ petroleum ether $): V($ EtOAc $)=$ $4: 1], 35.5 \mathrm{mg}, 72 \%$ yield. Yellow solid, m.p. 121.5 $122.1{ }^{\circ} \mathrm{C} ;{ }^{1} \mathrm{H}$ NMR $\left(400 \mathrm{MHz}, \mathrm{CDCl}_{3}\right) \delta: 7.25(\mathrm{t}, J=8.0$ $\mathrm{Hz}, 1 \mathrm{H}), 6.91 \sim 6.81(\mathrm{~m}, 3 \mathrm{H}), 5.78(\mathrm{~s}, 1 \mathrm{H}), 5.05(\mathrm{dd}, J=$ 8.0, $2.8 \mathrm{~Hz}, 1 \mathrm{H}), 4.78$ (brs, $1 \mathrm{H}), 4.15$ (dd, $J=14.0,3.2 \mathrm{~Hz}$, $1 \mathrm{H}), 4.01(\mathrm{dd}, J=14.0,8.0 \mathrm{~Hz}, 1 \mathrm{H}), 3.78$ (s, 3H), 2.24 (s, $3 \mathrm{H}), 2.02(\mathrm{~d}, J=0.4 \mathrm{~Hz}, 3 \mathrm{H}) ;{ }^{13} \mathrm{C} \mathrm{NMR}\left(100 \mathrm{MHz}, \mathrm{CDCl}_{3}\right)$ $\delta: 159.7,148.2,142.8,140.0,129.5,118.1,113.7,111.1$, 105.0, 73.4, 55.2, 54.8, 13.4, 10.7; HRMS (ESI-TOF) calcd for $\mathrm{C}_{14} \mathrm{H}_{19} \mathrm{~N}_{2} \mathrm{O}[\mathrm{M}+\mathrm{H}]^{+}$231.1492, found 231.1496.

2-(3,5-Dimethyl-1H-pyrazol-1-yl)-1-(3-fluorophenyl)ethanol (18): $R_{\mathrm{f}}=0.2[V($ petroleum ether $): V($ EtOAc $)=$ $4: 1], 41.7 \mathrm{mg}, 89 \%$ yield. White solid, m.p. 125.5 $127.8{ }^{\circ} \mathrm{C} ;{ }^{1} \mathrm{H}$ NMR $\left(400 \mathrm{MHz}, \mathrm{CDCl}_{3}\right) \delta: 7.33 \sim 7.27(\mathrm{~m}$, $1 \mathrm{H}), 7.08 \sim 6.95(\mathrm{~m}, 3 \mathrm{H}), 5.79(\mathrm{~s}, 1 \mathrm{H}), 5.08(\mathrm{dd}, J=7.6$, $2.8 \mathrm{~Hz}, 1 \mathrm{H}), 5.00$ (brs, $1 \mathrm{H}), 4.15$ (dd, $J=14.0,3.2 \mathrm{~Hz}$, $1 \mathrm{H}), 3.99$ (dd, $J=14.0,7.6 \mathrm{~Hz}, 1 \mathrm{H}), 2.24(\mathrm{~s}, 3 \mathrm{H}), 2.02$ (s, $3 \mathrm{H}) ;{ }^{13} \mathrm{C}$ NMR $\left(100 \mathrm{MHz}, \mathrm{CDCl}_{3}\right) \delta: 162.9(\mathrm{~d}, J=245.0$ $\mathrm{Hz}), 148.3,143.8$ (d, $J=6.9 \mathrm{~Hz}), 139.9,130.0$ (d, $J=8.1$ $\mathrm{Hz}), 121.4$ (d, $J=2.6 \mathrm{~Hz}), 114.6(\mathrm{~d}, J=21.2 \mathrm{~Hz}), 112.9$ (d, $J=22.0 \mathrm{~Hz}), 105.1,72.9,54.5,13.4,10.7 ;{ }^{19} \mathrm{~F}$ NMR $(376$ $\mathrm{MHz}, \mathrm{CDCl}_{3}$ ) $\delta$ : -112.8 ; HRMS (ESI-TOF) calcd for $\mathrm{C}_{13} \mathrm{H}_{15} \mathrm{FN}_{2} \mathrm{NaO}[\mathrm{M}+\mathrm{Na}]^{+}$257.1061, found 257.1068.

1-(3-Chlorophenyl)-2-(3,5-dimethyl-1 $H$-pyrazol-1-yl)ethanol (19): $R_{\mathrm{f}}=0.2[V($ petroleum ether $): V($ EtOAc $)=$ $4: 1], 45.1 \mathrm{mg}, 90 \%$ yield. White solid, m.p. 125.5 $127.3{ }^{\circ} \mathrm{C} ;{ }^{1} \mathrm{H}$ NMR (400 MHz, $\left.\mathrm{CDCl}_{3}\right) \delta: 7.32(\mathrm{~s}, 1 \mathrm{H})$, $7.27 \sim 7.26(\mathrm{~m}, 2 \mathrm{H}), 7.19 \sim 7.18(\mathrm{~m}, 1 \mathrm{H}), 5.79(\mathrm{~s}, 1 \mathrm{H})$, $5.05(\mathrm{dd}, J=7.6,2.8 \mathrm{~Hz}, 1 \mathrm{H}), 4.14(\mathrm{dd}, J=14.0,2.8 \mathrm{~Hz}$, $1 \mathrm{H}), 3.98$ (dd, $J=14.0,8.0 \mathrm{~Hz}, 1 \mathrm{H}), 2.24$ (s, 3H), 2.03 (s, $3 \mathrm{H}) ;{ }^{13} \mathrm{C}$ NMR $\left(100 \mathrm{MHz}, \mathrm{CDCl}_{3}\right) \delta: 148.4,143.2,139.9$, $134.4,129.7,128.0,126.2,124.1,105.1,72.9,54.5,13.4$, 10.7; HRMS (ESI-TOF) calcd for $\mathrm{C}_{13} \mathrm{H}_{16} \mathrm{ClN}_{2} \mathrm{O}[\mathrm{M}+\mathrm{H}]^{+}$ 251.0946, found 251.0950.

2-(3,5-Dimethyl-1H-pyrazol-1-yl)-1-(3-(trifluoromethoxy)phenyl)ethanol (20): $R_{\mathrm{f}}=0.2[V$ (petroleum ether) : $V($ EtOAc $)=4: 1], 42.6 \mathrm{mg}, 71 \%$ yield. White solid, m.p. $106.3 \sim 107.2{ }^{\circ} \mathrm{C} ;{ }^{1} \mathrm{H}$ NMR $\left(400 \mathrm{MHz}, \mathrm{CDCl}_{3}\right) \delta: 7.35$ (t, 
$J=7.6 \mathrm{~Hz}, 1 \mathrm{H}), 7.21$ (d, $J=7.6 \mathrm{~Hz}, 1 \mathrm{H}), 7.14$ (s, 1H), 7.12 $(\mathrm{d}, J=10.0 \mathrm{~Hz}, 1 \mathrm{H}), 5.77(\mathrm{~s}, 1 \mathrm{H}), 5.10(\mathrm{dd}, J=7.2,2.8 \mathrm{~Hz}$, $1 \mathrm{H}), 4.16(\mathrm{dd}, J=14.0,2.8 \mathrm{~Hz}, 1 \mathrm{H}), 4.01(\mathrm{dd}, J=14.0,7.2$ $\mathrm{Hz}, 1 \mathrm{H}), 2.23$ (s, 3H), 1.97 (s, 3H); ${ }^{13} \mathrm{C} \mathrm{NMR}(100 \mathrm{MHz}$, $\left.\mathrm{CDCl}_{3}\right) \delta: 149.4,148.4,143.7,140.0,129.8,124.2,120.4$ (d, $J=255.3 \mathrm{~Hz}), 120.2,118.5,105.1,72.8,54.4,13.3$, 10.6; ${ }^{19} \mathrm{~F} \mathrm{NMR}\left(376 \mathrm{MHz}, \mathrm{CDCl}_{3}\right) \delta$ : -57.7 ; HRMS (ESI-TOF) calcd for $\mathrm{C}_{14} \mathrm{H}_{15} \mathrm{~F}_{3} \mathrm{~N}_{2} \mathrm{NaO}_{2}[\mathrm{M}+\mathrm{Na}]^{+}$ 323.0978, found 323.0979.

2-(3,5-Dimethyl-1H-pyrazol-1-yl)-1-(3-(trifluoromethyl)phenyl)ethanol (21): $R_{\mathrm{f}}=0.2 \quad[V$ (petroleum ether) : $V($ EtOAc $)=4: 1], 44.5 \mathrm{mg}, 79 \%$ yield. White solid, m.p. 147.6 148.2 ${ }^{\circ} \mathrm{C} ;{ }^{1} \mathrm{H}$ NMR (400 $\left.\mathrm{MHz}, \mathrm{CDCl}_{3}\right) \delta$ : $7.54(\mathrm{~s}$, $2 \mathrm{H}), 7.50 \sim 7.43(\mathrm{~m}, 2 \mathrm{H}), 5.78(\mathrm{~s}, 1 \mathrm{H}), 5.14(\mathrm{dd}, J=7.6$, $2.8 \mathrm{~Hz}, 2 \mathrm{H}), 4.17(\mathrm{dd}, J=14.0,2.8 \mathrm{~Hz}, 1 \mathrm{H}), 4.01(\mathrm{dd}, J=$ 14.0, $8.0 \mathrm{~Hz}, 1 \mathrm{H}), 2.24(\mathrm{~s}, 3 \mathrm{H}), 1.99(\mathrm{~s}, 3 \mathrm{H}) ;{ }^{13} \mathrm{C} \mathrm{NMR}$ $\left(100 \mathrm{MHz}, \mathrm{CDCl}_{3}\right) \delta: 148.4,142.2,140.0,130.8$ (q, $J=$ $31.8 \mathrm{~Hz}), 129.3,128.9,124.6$ (q, $J=3.4 \mathrm{~Hz}), 124.0$ (q, $J=$ $271.5 \mathrm{~Hz}), 122.8$ (d, $J=3.6 \mathrm{~Hz}), 105.1,72.9,54.5,13.3$, 10.6; ${ }^{19} \mathrm{~F}$ NMR (376 MHz, $\mathrm{CDCl}_{3}$ ) $\delta$ : - 62.7; HRMS (ESI-TOF) calcd for $\mathrm{C}_{14} \mathrm{H}_{15} \mathrm{~F}_{3} \mathrm{~N}_{2} \mathrm{NaO} \quad[\mathrm{M}+\mathrm{Na}]^{+}$ 307.1029 , found 307.1037 .

2-(3,5-Dimethyl-1H-pyrazol-1-yl)-1-(3,4-dimethylphenyl)ethanol (22): $R_{\mathrm{f}}=0.2$ [ $V$ (petroleum ether) $: V($ EtOAc $)=$ 4:1], $39.6 \mathrm{mg}, 81 \%$ yield. White solid, m.p. 117.6 $118.3{ }^{\circ} \mathrm{C} ;{ }^{1} \mathrm{H}$ NMR $\left(400 \mathrm{MHz}, \mathrm{CDCl}_{3}\right) \delta: 7.11(\mathrm{~d}, J=7.6$ $\mathrm{Hz}, 2 \mathrm{H}), 7.06$ (dd, $J=7.8,1.6 \mathrm{~Hz}, 1 \mathrm{H}), 5.80$ (s, 1H), 5.02 $(\mathrm{dd}, J=8.4,2.8 \mathrm{~Hz}, 1 \mathrm{H}), 4.58(\mathrm{~s}, 1 \mathrm{H}), 4.11(\mathrm{dd}, J=13.6$, $2.8 \mathrm{~Hz}, 1 \mathrm{H}), 4.00$ (dd, $J=14.0,8.4 \mathrm{~Hz}, 1 \mathrm{H}), 2.26$ (s, 3H), $2.26(\mathrm{~s}, 3 \mathrm{H}), 2.25(\mathrm{~s}, 3 \mathrm{H}), 2.08(\mathrm{~s}, 3 \mathrm{H}) ;{ }^{13} \mathrm{C}$ NMR $(100$ $\left.\mathrm{MHz}, \mathrm{CDCl}_{3}\right) \delta: 148.1,139.8,138.5,136.7,136.2,129.7$, $127.2,123.3,105.0,73.4,55.0,19.8,19.4,13.4,10.8$; HRMS (ESI-TOF) calcd for $\mathrm{C}_{15} \mathrm{H}_{21} \mathrm{~N}_{2} \mathrm{O}[\mathrm{M}+\mathrm{H}]{ }^{+}$ 245.1648 , found 245.1656 .

1-(3,4-Difluorophenyl)-2-(3,5-dimethyl-1H-pyrazol-1yl)ethanol (23): $R_{\mathrm{f}}=0.2$ [ $V$ (petroleum ether) $: V($ EtOAc $)=$ 4:1], $47.4 \mathrm{mg}$, 94\% yield. White solid, m.p. 104.2 $106.1{ }^{\circ} \mathrm{C} ;{ }^{1} \mathrm{H}$ NMR $\left(400 \mathrm{MHz}, \mathrm{CDCl}_{3}\right) \delta: 7.16 \sim 7.09(\mathrm{~m}$, 2H), $7.02 \sim 7.00(\mathrm{~m}, 1 \mathrm{H}), 5.79(\mathrm{~s}, 1 \mathrm{H}), 5.06$ (brs, $1 \mathrm{H}), 5.04$ $(\mathrm{dd}, J=7.6,2.8 \mathrm{~Hz}, 1 \mathrm{H}), 4.12(\mathrm{dd}, J=14.0,3.2 \mathrm{~Hz}, 1 \mathrm{H})$, $3.96(\mathrm{dd}, J=14.0,7.6 \mathrm{~Hz}, 1 \mathrm{H}), 2.23$ (s, 3H), 2.03 (s, 3H); ${ }^{13} \mathrm{C}$ NMR $\left(100 \mathrm{MHz}, \mathrm{CDCl}_{3}\right) \delta: 150.4(\mathrm{dd}, J=247.2,12.7$ Hz), 149.9 (dd, $J=246.3,12.5 \mathrm{~Hz}), 148.4,139.9,138.3$ (t, $J=4.1 \mathrm{~Hz}), 121.8(\mathrm{dd}, J=6.2,3.6 \mathrm{~Hz}), 117.2(\mathrm{~d}, J=17.2$ $\mathrm{Hz}), 115.0$ (d, $J=17.8 \mathrm{~Hz}), 105.1,72.3,54.5,13.4,10.7$; ${ }^{19} \mathrm{~F}$ NMR $\left(376 \mathrm{MHz}, \mathrm{CDCl}_{3}\right) \delta$ : $-137.3,-139.1$; HRMS (ESI-TOF) calcd for $\mathrm{C}_{13} \mathrm{H}_{15} \mathrm{~F}_{2} \mathrm{~N}_{2} \mathrm{O}[\mathrm{M}+\mathrm{H}]^{+}$253.1147, found 253.1146 .

1-(3,4-Dichlorophenyl)-2-(3,5-dimethyl-1H-pyrazol-1yl)ethanol (24): $R_{\mathrm{f}}=0.2$ [ $V$ (petroleum ether) $: V($ EtOAc $)=$ $4: 1$ ], $47.3 \mathrm{mg}, 83 \%$ yield. Yellow solid, m.p. 128.3 $130.5{ }^{\circ} \mathrm{C} ;{ }^{1} \mathrm{H}$ NMR (400 MHz, $\left.\mathrm{CDCl}_{3}\right) \delta: 7.41(\mathrm{~s}, 1 \mathrm{H})$, $7.40(\mathrm{~d}, J=8.4 \mathrm{~Hz}, 1 \mathrm{H}), 7.13(\mathrm{~d}, J=8.0 \mathrm{~Hz}, 1 \mathrm{H}), 5.80$ (s, 1H), 5.14 (s, 1H),5.04 (dd, $J=7.6,2.8 \mathrm{~Hz}, 2 \mathrm{H}), 4.12$ (dd, $J=14.0,2.8 \mathrm{~Hz}, 1 \mathrm{H}), 3.96(\mathrm{dd}, J=14.0,8.0 \mathrm{~Hz}, 1 \mathrm{H}), 2.23$ $(\mathrm{s}, 3 \mathrm{H}), 2.04(\mathrm{~s}, 3 \mathrm{H}) ;{ }^{13} \mathrm{C} \mathrm{NMR}\left(100 \mathrm{MHz}, \mathrm{CDCl}_{3}\right) \delta$ : $148.5,141.4,139.9,132.6,131.7,130.4,128.0,125.3$,
105.2, 72.4, 54.3, 13.4, 10.8; HRMS (ESI-TOF) calcd for $\mathrm{C}_{13} \mathrm{H}_{15} \mathrm{Cl}_{2} \mathrm{~N}_{2} \mathrm{O}[\mathrm{M}+\mathrm{H}]^{+}$285.0556, found 285.0566.

1-(2,3-Dihydrobenzofuran-5-yl)-2-(3,5-dimethyl-1H-pyrazol-1-yl)ethanol (25): $R_{\mathrm{f}}=0.2[V($ petroleum ether) : $V($ EtOAc $)=3: 1], 42.4 \mathrm{mg}, 84 \%$ yield. White solid, m.p. 162.6 163.5 ${ }^{\circ} \mathrm{C} ;{ }^{1} \mathrm{H}$ NMR $\left(400 \mathrm{MHz}, \mathrm{CDCl}_{3}\right) \delta: 7.19$ (s, $1 \mathrm{H}), 7.05(\mathrm{~d}, J=8.4 \mathrm{~Hz}, 1 \mathrm{H}), 6.74(\mathrm{~d}, J=8.0 \mathrm{~Hz}, 1 \mathrm{H}), 5.79$ (s, 1H), 5.00 (dd, $J=8.4,2.8 \mathrm{~Hz}, 1 \mathrm{H}), 4.64(\mathrm{~s}, 1 \mathrm{H}), 4.57$ (t, $J=8.8 \mathrm{~Hz}, 2 \mathrm{H}), 4.09$ (dd, $J=13.6,2.8 \mathrm{~Hz}, 1 \mathrm{H}), 3.98$ (dd, $J=14.0,8.4 \mathrm{~Hz}, 1 \mathrm{H}), 3.19(\mathrm{t}, J=8.8 \mathrm{~Hz}, 2 \mathrm{H}), 2.24(\mathrm{~s}, 3 \mathrm{H})$, $2.07(\mathrm{~s}, 3 \mathrm{H}) ;{ }^{13} \mathrm{C}$ NMR $\left(100 \mathrm{MHz}, \mathrm{CDCl}_{3}\right) \delta: 159.9,148.1$, 139.8, 133.2, 127.3, 125.9, 122.6, 109.1, 105.0, 73.4, 71.3, 55.1, 29.7, 13.4, 10.8; HRMS (ESI-TOF) calcd for $\mathrm{C}_{15} \mathrm{H}_{19}$ $\mathrm{N}_{2} \mathrm{O}_{2}[\mathrm{M}+\mathrm{H}]^{+}$259.1441, found 259.1447.

1-(Benzo[d][1,3]dioxol-5-yl)-2-(3,5-dimethyl-1H-pyrazol-1-yl)ethanol (26): $R_{\mathrm{f}}=0.2 \quad[V$ (petroleum ether) : $V($ EtOAc $)=2: 1], 46.3 \mathrm{mg}, 89 \%$ yield. White solid, m.p. 158.2 160.1 ${ }^{\circ} \mathrm{C} ;{ }^{1} \mathrm{H}$ NMR (400 MHz, $\left.\mathrm{CDCl}_{3}\right) \delta: 6.82(\mathrm{~s}$, $1 \mathrm{H}), 6.78(\mathrm{~s}, 2 \mathrm{H}), 5.95(\mathrm{~s}, 2 \mathrm{H}), 5.79(\mathrm{~s}, 1 \mathrm{H}), 4.99$ (dd, $J=$ 8.0, $2.4 \mathrm{~Hz}, 1 \mathrm{H}), 4.74$ (s, 1H), 4.09 (dd, $J=14.0,3.2 \mathrm{~Hz}$, $1 \mathrm{H}), 3.97$ (dd, $J=14.0,8.0 \mathrm{~Hz}, 1 \mathrm{H}), 2.24(\mathrm{~s}, 3 \mathrm{H}), 2.06$ (s, $3 \mathrm{H}) ;{ }^{13} \mathrm{C} \mathrm{NMR}\left(100 \mathrm{MHz}, \mathrm{CDCl}_{3}\right) \delta: 148.2,147.8,147.2$, 139.8, 135.1, 119.3, 108.2, 106.5, 105.0, 101.0, 73.3, 54.9, 13.4, 10.8; HRMS (ESI-TOF) calcd for $\mathrm{C}_{14} \mathrm{H}_{16} \mathrm{~N}_{2} \mathrm{NaO}_{3}$ $[\mathrm{M}+\mathrm{Na}]^{+}$283.1053, found 283.1047.

1-(3,5-Difluorophenyl)-2-(3,5-dimethyl-1 $H$-pyrazol-1yl)ethanol (27): $R_{\mathrm{f}}=0.2$ [ $V$ (petroleum ether) $: V($ EtOAc $)=$ $4: 1], 31.8 \mathrm{mg}, 63 \%$ yield. White solid, m.p. 113.6 $114.8{ }^{\circ} \mathrm{C} ;{ }^{1} \mathrm{H}$ NMR $\left(400 \mathrm{MHz}, \mathrm{CDCl}_{3}\right) \delta: 6.85 \sim 6.83(\mathrm{~m}$, $1 \mathrm{H}), 6.74 \sim 6.69(\mathrm{~m}, 1 \mathrm{H}), 5.79(\mathrm{~s}, 1 \mathrm{H}), 5.21(\mathrm{~s}, 1 \mathrm{H}), 5.04$ (dd, $J=7.6,2.8 \mathrm{~Hz}, 1 \mathrm{H}), 4.13$ (dd, $J=14.0,3.2 \mathrm{~Hz}, 1 \mathrm{H})$, 3.97 (dd, $J=14.0,7.6 \mathrm{~Hz}, 1 \mathrm{H}), 2.22$ (s, 3H), 2.04 (s, 3H); ${ }^{13} \mathrm{C}$ NMR $\left(100 \mathrm{MHz}, \mathrm{CDCl}_{3}\right) \delta: 163.1(\mathrm{dd}, J=247.5,12.4$ $\mathrm{Hz}), 148.5,145.4$ (t, $J=8.5 \mathrm{~Hz}), 139.9,108.8$ (d, $J=25.6$, $7.1 \mathrm{~Hz}), 105.2,103.1(\mathrm{t}, J=25.1 \mathrm{~Hz}), 72.6,54.2,13.4$, 10.7; ${ }^{19} \mathrm{~F}$ NMR (377 MHz, $\left.\mathrm{CDCl}_{3}\right) \delta$ : -109.3 ; HRMS (ESI-TOF) calcd for $\mathrm{C}_{13} \mathrm{H}_{15} \mathrm{~F}_{2} \mathrm{~N}_{2} \mathrm{O}[\mathrm{M}+\mathrm{H}]^{+}$253.1147, found 253.1146 .

1-(3,5-Dichlorophenyl)-2-(3,5-dimethyl-1H-pyrazol-1yl)ethanol (28): $R_{\mathrm{f}}=0.2$ [ $V$ (petroleum ether) $: V($ EtOAc $)=$ 4:1], $31.9 \mathrm{mg}, 56 \%$ yield. White solid, m.p. 140.5 $141.2{ }^{\circ} \mathrm{C} ;{ }^{1} \mathrm{H}$ NMR $\left(400 \mathrm{MHz}, \mathrm{CDCl}_{3}\right) \delta: 7.28(\mathrm{t}, J=2.0$ $\mathrm{Hz}, 1 \mathrm{H}), 7.20$ (d, $J=2.0 \mathrm{~Hz}, 2 \mathrm{H}), 5.80(\mathrm{~s}, 1 \mathrm{H}), 5.23(\mathrm{~s}$, $1 \mathrm{H}), 5.02$ (dd, $J=7.6,3.2 \mathrm{~Hz}, 1 \mathrm{H}), 4.12(\mathrm{dd}, J=14.0,2.8$ $\mathrm{Hz}, 1 \mathrm{H}), 3.96$ (dd, $J=14.0,7.6 \mathrm{~Hz}, 1 \mathrm{H}), 2.23$ (s, 3H), 2.05 $(\mathrm{s}, 3 \mathrm{H}) ;{ }^{13} \mathrm{C}$ NMR $\left(100 \mathrm{MHz}, \mathrm{CDCl}_{3}\right) \delta: 148.5,144.6$, $139.9,135.1,127.9,124.5,105.2,72.6,54.2,13.4,10.8$; HRMS (ESI-TOF) calcd for $\mathrm{C}_{13} \mathrm{H}_{15} \mathrm{Cl}_{2} \mathrm{~N}_{2} \mathrm{O}[\mathrm{M}+\mathrm{H}]^{+}$ 285.0556, found 285.0562 .

2-(3,5-Dimethyl-1H-pyrazol-1-yl)-1-(naphthalen-2-yl)ethanol (29): $R_{\mathrm{f}}=0.2[V($ petroleum ether $): V($ EtOAc $)=$ 4:1], $37.3 \mathrm{mg}, 70 \%$ yield. White solid, m.p. 139.8 $141.7{ }^{\circ} \mathrm{C} ;{ }^{1} \mathrm{H}$ NMR $\left(400 \mathrm{MHz}, \mathrm{CDCl}_{3}\right) \delta: 7.84 \sim 7.81(\mathrm{~m}$, $4 \mathrm{H}), 7.49 \sim 7.46(\mathrm{~m}, 2 \mathrm{H}), 7.42(\mathrm{dd}, J=8.4,1.6 \mathrm{~Hz}, 1 \mathrm{H})$, 5.79 (s, 1H), 5.25 (dd, $J=7.6,2.8 \mathrm{~Hz}, 1 \mathrm{H}), 4.89$ (brs, 1H), 4.24 (dd, $J=13.6,2.8 \mathrm{~Hz}, 1 \mathrm{H}), 4.10$ (dd, $J=14.0,8.0 \mathrm{~Hz}$, 1H), $2.27(\mathrm{~s}, 3 \mathrm{H}), 2.02(\mathrm{~s}, 3 \mathrm{H}) ;{ }^{13} \mathrm{C} \mathrm{NMR}(100 \mathrm{MHz}$, 
$\left.\mathrm{CDCl}_{3}\right) \delta: 148.2,139.9,138.5,133.3,133.1,128.2,128.0$ $127.7,126.2,125.9,124.9,123.8,105.3,73.6,54.8,13.4$, 10.8; HRMS (ESI-TOF) calcd for $\mathrm{C}_{17} \mathrm{H}_{18} \mathrm{~N}_{2} \mathrm{NaO}[\mathrm{M}+$ $\mathrm{Na}]^{+}$289.1311, found 289.1301.

2-(3,5-Dimethyl-1H-pyrazol-1-yl)-1-(naphthalen-1-yl)ethanol (30): $R_{\mathrm{f}}=0.2[V($ petroleum ether $): V($ EtOAc $)=$ $4: 1], 42.6 \mathrm{mg}, 80 \%$ yield. White solid, m.p. 138.0 $140.0{ }^{\circ} \mathrm{C} ;{ }^{1} \mathrm{H}$ NMR (400 MHz, $\left.\mathrm{CDCl}_{3}\right) \delta: 8.08$ (d, $J=8.4$ $\mathrm{Hz}, 1 \mathrm{H}), 7.91 \sim 7.89(\mathrm{~m}, 1 \mathrm{H}), 7.81(\mathrm{~d}, J=8.0 \mathrm{~Hz}, 1 \mathrm{H})$, $7.68(\mathrm{~d}, J=7.2 \mathrm{~Hz}, 1 \mathrm{H}), 7.56 \sim 7.46(\mathrm{~m}, 3 \mathrm{H}), 5.88(\mathrm{~d}, J=$ $6.0 \mathrm{~Hz}, 1 \mathrm{H}), 5.79$ (s, 1H), 5.09 (brs, 1H), 4.37 (dd, $J=$ $14.0,2.8 \mathrm{~Hz}, 1 \mathrm{H}), 4.14$ (dd, $J=14.0,7.6 \mathrm{~Hz}, 1 \mathrm{H}), 2.28$ (s, $3 \mathrm{H}), 1.94(\mathrm{~s}, 3 \mathrm{H}) ;{ }^{13} \mathrm{C} \mathrm{NMR}\left(100 \mathrm{MHz}, \mathrm{CDCl}_{3}\right) \delta: 148.3$, $139.9,136.4,133.6,130.1,129.1,128.2,126.3,125.6$, 125.5, 123.7, 122.3, 105.0, 70.3, 54.0, 13.5, 10.7; HRMS (ESI-TOF) calcd for $\mathrm{C}_{17} \mathrm{H}_{19} \mathrm{~N}_{2} \mathrm{O}[\mathrm{M}+\mathrm{H}]^{+}$267.1492, found 267.1491 .

2-(3,5-Dimethyl-1H-pyrazol-1-yl)-1-(1-methyl-1H-indol-3-yl)ethanol (31): $R_{\mathrm{f}}=0.2 \quad[V$ (petroleum ether) : $V($ EtOAc $)=2: 1], 39.3 \mathrm{mg}, 73 \%$ yield. White solid, m.p. 123.4 126.4 ${ }^{\circ} \mathrm{C} ;{ }^{1} \mathrm{H}$ NMR (400 MHz, $\left.\mathrm{CDCl}_{3}\right) \delta: 7.69(\mathrm{~d}$, $J=8.0 \mathrm{~Hz}, 1 \mathrm{H}), 7.26(\mathrm{t}, J=8.0 \mathrm{~Hz}, 1 \mathrm{H}), 7.14(\mathrm{t}, J=8.0$ $\mathrm{Hz}, 1 \mathrm{H}), 7.06$ (s, 1H), $5.82(\mathrm{~s}, 1 \mathrm{H}), 5.43$ (dd, $J=8.4,2.8$ $\mathrm{Hz}, 1 \mathrm{H}), 4.29$ (dd, $J=14.0,2.8 \mathrm{~Hz}, 1 \mathrm{H}), 4.23$ (dd, $J=14.0$, $8.4 \mathrm{~Hz}, 1 \mathrm{H}), 3.77$ (s, 3H), $2.26(\mathrm{~s}, 3 \mathrm{H}), 2.15(\mathrm{~s}, 3 \mathrm{H}) ;{ }^{13} \mathrm{C}$ NMR $\left(100 \mathrm{MHz}, \mathrm{CDCl}_{3}\right) \delta: 148.0,139.7,137.2,126.6$, $125.9,121.8,119.3,119.1,114.7,109.5,104.9,68.1,54.3$, 32.7, 13.5, 11.0; HRMS (ESI-TOF) calcd for $\mathrm{C}_{16} \mathrm{H}_{20} \mathrm{~N}_{3} \mathrm{O}$ $[\mathrm{M}+\mathrm{H}]^{+}$270.1601, found 270.1606 .

\subsection{Procedures for the synthesis of compound $\mathbf{3 8}$}

The synthesis of compound $\mathbf{3 8}$ was adapted from the literature procedures. ${ }^{[20]}$ To a $25 \mathrm{~mL}$ flask charged with crude 2 (0.2 mmol), $\mathrm{MeOH}(2.5 \mathrm{~mL})$ and $\mathrm{H}_{2} \mathrm{O}(2.5 \mathrm{~mL})$ was added $\mathrm{KHF}_{2}(78.0 \mathrm{mg}, 1.0 \mathrm{mmol})$ at room temperature. The resulting mixture was then allowed to stir at room temperature for $2 \mathrm{~h}$. After removal of the solvent, the residue was diluted by $\mathrm{H}_{2} \mathrm{O}(10 \mathrm{~mL})$ and extracted with EtOAc $(10 \mathrm{~mL} \times 3)$. The combined organic phase was dried over $\mathrm{Na}_{2} \mathrm{SO}_{4}$. After removal of the solvent, the residue was purified by column chromatography on silica gel using EtOAc as eluent to afford 1-(2-(difluoroboryl)-2-phenylethyl)-3,5-dimethyl- $1 H$-pyrazole (38). $R_{\mathrm{f}}=0.1$ (EtOAc), $34.7 \mathrm{mg}, 70 \%$ yield. White solid, m.p. $280.3 \sim 282.5{ }^{\circ} \mathrm{C}$; ${ }^{1} \mathrm{H}$ NMR (400 MHz, $d_{6}$-Acetone) $\delta: 7.21(\mathrm{t}, J=7.6 \mathrm{~Hz}$, $2 \mathrm{H}), 7.13(\mathrm{~d}, J=7.6 \mathrm{~Hz}, 2 \mathrm{H}), 7.08(\mathrm{t}, J=7.2 \mathrm{~Hz}, 1 \mathrm{H}), 6.25$ (s, $1 \mathrm{H}), 4.59$ (dd, $J=11.2,9.2 \mathrm{~Hz}, 1 \mathrm{H}), 4.19$ (dd, $J=11.6$, $7.2 \mathrm{~Hz}, 1 \mathrm{H}), 2.90 \sim 2.80(\mathrm{~m}, 1 \mathrm{H}), 2.37(\mathrm{~s}, 3 \mathrm{H}), 2.31(\mathrm{~s}$, $3 \mathrm{H}) ;{ }^{13} \mathrm{C}$ NMR (100 MHz, $d_{6}$-Acetone) $\delta: 144.2,142.4$, $140.7,128.7,128.3,125.3,109.7,53.0,40.0$ (br), 10.5, 10.1; ${ }^{11} \mathrm{~B}$ NMR (128 MHz, $d_{6}$-Acetone) $\delta: 6.95(\mathrm{t}, J=47.1$ $\mathrm{Hz}) ;{ }^{19} \mathrm{~F}$ NMR (376 MHz, $d_{6}$-Acetone) $\delta:-143.8$, 144.0; HRMS (ESI-TOF) calcd for $\mathrm{C}_{13} \mathrm{H}_{16} \mathrm{BF}_{2} \mathrm{~N}_{2}[\mathrm{M}+\mathrm{H}]^{+}$ 249.1369, found 249.1380.

\subsection{Procedures for the synthesis of compound $\mathbf{3 9}$}

The preparation of $\mathbf{3 9}$ was adapted from literature procedures. ${ }^{[21]}$ To a $25 \mathrm{~mL}$ flame-dried Schlenk tube charged with crude $2(0.20 \mathrm{mmol}), \mathrm{AgNO}_{3}(3.4 \mathrm{mg}, 0.2 \mathrm{~mol} \%$ ), Selectfluor (106 mg, $0.60 \mathrm{mmol}, 3.0$ equiv.), tetrahydrofuran (THF) $(0.5 \mathrm{~mL}), \mathrm{H}_{2} \mathrm{O}(0.5 \mathrm{~mL})$ and TFA $(31 \mu \mathrm{L}, 0.8$ mmol, 4.0 equiv.) were then added. The reaction mixture was allowed to stir at $60{ }^{\circ} \mathrm{C}$ for $6 \mathrm{~h}$. After cooling to room temperature, the reaction was quenched by slow addition of saturated aq. $\mathrm{NaHCO}_{3}(5 \mathrm{~mL})$, and the resulting mixture was extracted with EtOAc $(10 \mathrm{~mL} \times 3)$. The combined ethereal solution was dried over anhydrous $\mathrm{MgSO}_{4}$. After removal of the solvent, the residue was purified by column chromatography on silica using petroleum ether/EtOAc $(V: V=8: 1)$ as the eluent to afford 1-(2-fluoro2-phenylethyl)-3,5-dimethyl-1H-pyrazole (39). $\quad R_{\mathrm{f}}=0.5$ $[V($ petroleum ether $): V($ EtOAc $)=4: 1], 31.8 \mathrm{mg}, 73 \%$ yield. Yellow oil. ${ }^{1} \mathrm{H}$ NMR $\left(400 \mathrm{MHz}, \mathrm{CDCl}_{3}\right) \delta: 7.42 \sim$ $7.32(\mathrm{~m}, 5 \mathrm{H}), 5.92 \sim 5.75(\mathrm{~m}, 2 \mathrm{H}), 4.39 \sim 4.16(\mathrm{~m}, 2 \mathrm{H})$, $2.25(\mathrm{~s}, 3 \mathrm{H}), 2.13(\mathrm{~s}, 3 \mathrm{H}) ;{ }^{13} \mathrm{C} \mathrm{NMR}\left(100 \mathrm{MHz}, \mathrm{CDCl}_{3}\right) \delta$ : 148.2, 140.3, 137.0 (d, $J=19.4 \mathrm{~Hz}), 128.9$ (d, $J=1.0$ $\mathrm{Hz}), 125.4(\mathrm{~d}, J=6.9 \mathrm{~Hz}), 105.1,93.0(\mathrm{~d}, J=175.9 \mathrm{~Hz})$, $54.1(\mathrm{~d}, J=26.1 \mathrm{~Hz}), 13.4,10.8 ;{ }^{19} \mathrm{~F}$ NMR $(376 \mathrm{MHz}$, $\left.\mathrm{CDCl}_{3}\right) \quad \delta: \quad-183.0$; HRMS (ESI-TOF) calcd for $\mathrm{C}_{13} \mathrm{H}_{16} \mathrm{FN}_{2}[\mathrm{M}+\mathrm{H}]^{+}$219.1292, found 219.1292.

\subsection{Procedures for the synthesis of compound $\mathbf{4 0}$}

This reaction was adapted from the literature procedures. ${ }^{[22]}$ To a $25 \mathrm{~mL}$ flame-dried Schlenk tube charged with crude $2(0.2 \mathrm{mmol}), \mathrm{ClCH}_{2} \mathrm{I}(75 \mu \mathrm{L}, 1.0 \mathrm{mmol})$ and THF $(2 \mathrm{~mL})$ was added $n$-BuLi $\left(0.4 \mathrm{~mL}, 2.5 \mathrm{~mol} \cdot \mathrm{L}^{-1}\right.$ in hexanes, $1.0 \mathrm{mmol}$ ) at $-78{ }^{\circ} \mathrm{C}$ under $\mathrm{N}_{2}$ atmosphere. The resulting mixture was allowed to stir at the same temperature for $10 \mathrm{~min}$ and then allowed to stir at room temperature for $1 \mathrm{~h}$. The mixture was then diluted by $\mathrm{H}_{2} \mathrm{O}(10$ $\mathrm{mL})$. The biphasic mixture was then extracted by EtOAc $(10 \mathrm{~mL} \times 3)$. After removal of the solvent, $\mathrm{NaBO}_{3} \bullet 4 \mathrm{H}_{2} \mathrm{O}$ $(0.15 \mathrm{~g}, 1.0 \mathrm{mmol})$ in $\mathrm{THF} / \mathrm{H}_{2} \mathrm{O}(V: V=1: 1,4 \mathrm{~mL})$ was introduced to the above reaction mixture. The resulting biphasic mixture was then continued to stir at room temperature for $4 \mathrm{~h}$. The mixture was then diluted by $\mathrm{H}_{2} \mathrm{O}(10$ $\mathrm{mL})$ and quenched by saturated aqueous $\mathrm{Na}_{2} \mathrm{SO}_{3}(1 \mathrm{~mL})$. The biphasic mixture was then extracted by EtOAc $(10 \mathrm{~mL}$ $\times 3$ ). The combined organic phase was dried over $\mathrm{Na}_{2} \mathrm{SO}_{4}$. After removal of the solvent, the residue was purified by column chromatography on silica gel using EtOAc/petroleum ether $(V: V=3: 1)$ as the eluent to afford $3-(3,5$ dimethyl-1 $H$-pyrazol-1-yl)-2-phenylpropan-1-ol (40). $R_{\mathrm{f}}=$ $0.3[V$ (petroleum ether) $: V($ EtOAc $)=4: 1], 32.5 \mathrm{mg}$, $71 \%$ yield. White solid, m.p. 91.0 92.6 ${ }^{\circ} \mathrm{C} ;{ }^{1} \mathrm{H}$ NMR $\left(400 \mathrm{MHz}, \mathrm{CDCl}_{3}\right) \delta: 7.32 \sim 7.24(\mathrm{~m}, 3 \mathrm{H}), 7.14(\mathrm{~d}, J=7.2$ $\mathrm{Hz}, 2 \mathrm{H}), 5.74(\mathrm{~s}, 1 \mathrm{H}), 4.33 \sim 4.21(\mathrm{~m}, 2 \mathrm{H}), 3.87(\mathrm{dd}, J=$ 11.6, $6.8 \mathrm{~Hz}, 1 \mathrm{H}), 3.75(\mathrm{dd}, J=11.6,5.2 \mathrm{~Hz}, 1 \mathrm{H}), 3.22(\mathrm{dt}$, $J=12.0,6.0 \mathrm{~Hz}, 1 \mathrm{H}), 2.22(\mathrm{~s}, 3 \mathrm{H}), 1.91(\mathrm{~s}, 3 \mathrm{H}) ;{ }^{13} \mathrm{C} \mathrm{NMR}$ $\left(100 \mathrm{MHz}, \mathrm{CDCl}_{3}\right) \delta: 147.8,140.4,139.9,128.7,128.0$, 127.2, 104.9, 50.7, 48.4, 24.8, 13.4, 10.7; HRMS (ESITOF) calcd for $\mathrm{C}_{14} \mathrm{H}_{19} \mathrm{~N}_{2} \mathrm{O}[\mathrm{M}+\mathrm{H}]^{+}$231.1492, found 231.1499 .

\subsection{Procedures for the synthesis of compound $\mathbf{4 1}$}

This reaction was adapted from literature procedures. ${ }^{[23]}$ 
To a $25 \mathrm{~mL}$ flame-dried Schlenk tube charged with thiophene (42.2 mg, $0.5 \mathrm{mmol})$ and THF $(1 \mathrm{~mL})$ was added $n$-BuLi $\left(0.20 \mathrm{~mL}, 2.5 \mathrm{~mol} \cdot \mathrm{L}^{-1}\right.$ in hexanes, $\left.0.5 \mathrm{mmol}\right)$ at $78{ }^{\circ} \mathrm{C}$ under $\mathrm{N}_{2}$ atmosphere. The resulting mixture was allowed to stir at the same temperature for $2 \mathrm{~h}$. A THF (1 $\mathrm{mL})$ solution of curde $2(0.20 \mathrm{mmol})$ was then added at $-78{ }^{\circ} \mathrm{C}$. The resulting mixture was then allowed to stir at $-78{ }^{\circ} \mathrm{C}$ for $2 \mathrm{~h}$. A THF $(1 \mathrm{~mL})$ solution of $N$-bromosuccinimide (NBS) $(89.0 \mathrm{mg}, 0.5 \mathrm{mmol})$ was then added at room temperature. The reaction was continued to stir at the same temperature for $30 \mathrm{~min}$ then room temperature for 2 h. The mixture was diluted by $\mathrm{H}_{2} \mathrm{O}(10 \mathrm{~mL})$. The biphasic mixture was then extracted by $\mathrm{CH}_{2} \mathrm{Cl}_{2}$ three times (10 $\mathrm{mL} \times 3)$. The combined organic phase was dried over anhydrous $\mathrm{Na}_{2} \mathrm{SO}_{4}$. After removal of the solvent, the residue was purified by column chromatography on silica gel using EtOAc/petroleum ether $(V: V=8: 1)$ as eluent to afford 3,5-dimethyl-1-(2-phenyl-2-(thiophen-2-yl)ethyl)- $1 H$ - pyrazole $(\mathbf{4 1}) . R_{\mathrm{f}}=0.6[V$ (petroleum ether) $: V($ EtOAc $)=4$ : 1], $34.5 \mathrm{mg}, 61 \%$ yield. Yellow oil. ${ }^{1} \mathrm{H}$ NMR (400 MHz, $\left.\mathrm{CDCl}_{3}\right) \delta: 7.26 \sim 7.21(\mathrm{~m}, 3 \mathrm{H}), 7.18 \sim 7.16(\mathrm{~m}, 3 \mathrm{H}), 6.92$ $(\mathrm{t}, J=3.6 \mathrm{~Hz}, 1 \mathrm{H}), 6.74(\mathrm{~s}, 1 \mathrm{H}), 5.62(\mathrm{~s}, 1 \mathrm{H}), 4.92(\mathrm{t}, J=$ $7.6 \mathrm{~Hz}, 1 \mathrm{H}), 4.53(\mathrm{dd}, J=13.6,7.2 \mathrm{~Hz}, 1 \mathrm{H}), 4.36(\mathrm{dd}, J=$ 13.6, $8.0 \mathrm{~Hz}, 1 \mathrm{H}), 2.24(\mathrm{~s}, 3 \mathrm{H}), 1.67(\mathrm{~s}, 3 \mathrm{H}) ;{ }^{13} \mathrm{C} \mathrm{NMR}$ $\left(100 \mathrm{MHz}, \mathrm{CDCl}_{3}\right) \delta: 147.9,144.8,141.3,140.0,128.5$, $128.0,127.3,126.7,125.0,124.2,104.4,54.2,47.3,13.6$, 10.4; HRMS (ESI-TOF) calcd for $\mathrm{C}_{17} \mathrm{H}_{19} \mathrm{~N}_{2} \mathrm{~S} \quad[\mathrm{M}+\mathrm{H}]^{+}$ 283.1263 , found 283.1264 .

\subsection{Procedures for the synthesis of compound $\mathbf{4 2}$}

This reaction was adapted from literature procedures. ${ }^{[14 a]}$ To a $50 \mathrm{~mL}$ flame-dried flask charged with $39(115 \mathrm{mg}$, $0.5 \mathrm{mmol})$, pyridine $(0.5 \mathrm{~mL})$, and $\mathrm{MeOAc}(10 \mathrm{~mL})$ was bubbled ozone at $-78{ }^{\circ} \mathrm{C}$ for $1 \mathrm{~h}$. Ozone was replaced by nitrogen for 10 min bubbling. After that, $\mathrm{NiCl}_{2} \cdot 6 \mathrm{H}_{2} \mathrm{O}(0.12$ g, $0.5 \mathrm{mmol}), \mathrm{NaBH}_{4}(0.90 \mathrm{~g}, 2.5 \mathrm{mmol})$, and $\mathrm{MeOH}(5$ $\mathrm{mL}$ ) were successively added at the same temperature. The mixture was then allowed to warm to room temperature. Next two portions of $\mathrm{NaBH}_{4}(0.90 \mathrm{~g}, 2.5 \mathrm{mmol}$ each) were added after 1 and $6 \mathrm{~h}$, respectively. The mixture was then allowed to stir at room temperature for $12 \mathrm{~h}$, followed by slow addition of a saturated aqueous solution of $\mathrm{NaHCO}_{3}$ $(10 \mathrm{~mL})$ at $0{ }^{\circ} \mathrm{C}$. The mixture was then extracted by $\mathrm{CH}_{2} \mathrm{Cl}_{2}(20 \mathrm{~mL} \times 3)$. The combined organic phase was dried over anhydrous $\mathrm{Na}_{2} \mathrm{SO}_{4}$. After removal of the solvent, the residue was purified by column chromatography on silica gel using EtOAc/petroleum ether $(V: V=8: 1)$ as eluent to afford $N$-(2-fluoro-2-phenylethyl)acetamide (42). $R_{\mathrm{f}}=0.6[V$ (petroleum ether) $: V($ EtOAc $)=4: 1]$, $36.2 \mathrm{mg}, 40 \%$ yield. Yellow oil. ${ }^{1} \mathrm{H}$ NMR (400 MHz, $\left.\mathrm{CDCl}_{3}\right) \delta: 7.37 \sim 7.26(\mathrm{~m}, 5 \mathrm{H}), 5.99$ (brs, $\left.1 \mathrm{H}\right), 5.62 \sim 5.47$ $(\mathrm{m}, 1 \mathrm{H}), 4.00 \sim 3.85(\mathrm{~m}, 1 \mathrm{H}), 3.45 \sim 3.33(\mathrm{~m}, 1 \mathrm{H}), 2.03(\mathrm{~s}$, $1.4 \mathrm{H})^{*}, 2.02(\mathrm{~s}, 1.6 \mathrm{H})^{*}$ (*two Me signals due to of restriction $\mathrm{C}-\mathrm{N}$ bond rotation); ${ }^{13} \mathrm{C} \mathrm{NMR}(100 \mathrm{MHz}$, $\left.\mathrm{CDCl}_{3}\right) \delta: 170.3,137.1(\mathrm{~d}, J=18.6 \mathrm{~Hz}), 128.8,128.6$, 125.4 (d, $J=7.1 \mathrm{~Hz}), 93.0$ (d, $J=171.1 \mathrm{~Hz}), 45.1$ (d, $J=$ $23.4 \mathrm{~Hz}), 23.2 ;{ }^{19} \mathrm{~F}$ NMR $\left(376 \mathrm{MHz}, \mathrm{CDCl}_{3}\right) \delta$ : -183.3 ;
HRMS (EI-TOF) calcd for $\mathrm{C}_{10} \mathrm{H}_{12} \mathrm{FNO} 181.0897$, found 181.0895 .

\section{References}

[1] (a) Cho, J.-Y.; Tse, M. K.; Holmes, D.; Maleczka, R. E.; Smith, M. R. Science 2002, 295, 305.

(b) Ishiyama, T.; Takagi, J.; Ishida, K.; Miyaura, N.; Anastasi, N. R.; Hartwig, J. F. J. Am. Chem. Soc. 2002, 124, 390.

(c) Mkhalid, I. A. I.; Barnard, J. H.; Marder, T. B.; Murphy, J. M.; Hartwig, J. F. Chem. Rev. 2010, 110, 890. (d) Wang, M.; Shi, Z. Chem. Rev. 2020, 120, 7438.

[2] Boller, T. M.; Murphy, J. M.; Hapke, M.; Ishiyama, T.; Miyaura, N.; Hartwig, J. F. J. Am. Chem. Soc. 2005, 127, 14263.

[3] (a) Ros, A.; Fernández, R.; Lassaletta, J. M. Chem. Soc. Rev. 2014, 43, 3229.

(b) Hartwig, J. F. Chem. Soc. Rev. 2011, 40, 1992.

(c) Xu, L.; Wang, G.; Zhang, S.; Wang, H.; Wang, L.; Liu, L.; Jiao, J.; Li, P. Tetrahedron 2017, 73, 7123.

(d) Jin, J.; Xia, H.; Zhang, F.; Wang, Y. Chin. J. Org. Chem. 2020, 40, 2185 (in Chinese).

(靳继康, 夏慧敏, 张凤莲, 汪义丰, 有机化学, 2020, 40, 2185.)

(e) Shi, D.; Wang, L.; Xia, C.; Liu, C. Chin. J. Org. Chem. 2020, 40, 3605 (in Chinese).

(史敦发, 王露, 夏春谷, 刘超, 有机化学, 2020, 40, 3605.)

(f) He, H.; Jiang, Z. Chin. J. Org. Chem. 2020, 40, 3483 (in Chinese).

(许荷欢, 江智勇, 有机化学, 2020, 40, 3483.)

(g) Zhu, S.; Chu, L. Chin. J. Org. Chem. 2020, 40, 3980 (in Chinese).

(朱圣卿, 储玲玲, 有机化学, 2020, 40, 3980.)

[4] Selected examples, see:

(a) Thongpaen, J.; Schmid, T. E.; Toupet, L.; Dorcet, V.; Mauduit, M.; Baslé, O. Chem. Commun. 2018, 54, 8202.

(b) Roering, A. J.; Hale, L. V. A.; Squier, P. A.; Ringgold, M. A.; Wiederspan, E. R.; Clark, T. B. Org. Lett. 2012, 14, 3558.

(c) Xu, F.; Duke, O. M.; Rojas, D.; Eichelberger, H. M.; Kim, R. S.; Clark, T. B.; Watson, D. A. J. Am. Chem. Soc. 2020, 142, 11988.

(d) Nakamura, T.; Suzuki, K.; Yamashita, M. J. Am. Chem. Soc. 2017, 139, 17763.

(e) Wang, G.; Liu, L.; Wang, H.; Ding, Y.-S.; Zhou, J.; Mao, S.; Li, P. J. Am. Chem. Soc. 2017, 139, 91.

(f) Ghaffari, B.; Preshlock, S. M.; Plattner, D. L.; Staples, R. J.; Maligres, P. E.; Krska, S. W.; Maleczka, R. E.; Smith, M. R. J. Am. Chem. Soc. 2014, 136, 14345.

(g) Wright, S. E.; Richardson-Solorzano, S.; Stewart, T. N.; Miller, C. D.; Morris, K. C.; Daley, C. J. A.; Clark, T. B. Angew. Chem. Int. Ed. 2019, 58, 2834.

(h) Kawamorita, S.; Miyazaki, T.; Ohmiya, H.; Iwai, T.; Sawamura, M. J. Am. Chem. Soc. 2011, 133, 19310.

(i) Kawamorita, S.; Ohmiya, H.; Hara, K.; Fukuoka, A.; Sawamura, M. J. Am. Chem. Soc. 2009, 131, 5058.

(j) Ros, A.; Estepa, B.; López-Rodríguez, R.; Álvarez, E.; Fernández, R.; Lassaletta, J. M. Angew. Chem. Int. Ed. 2011, 50, 11724.

(k) Ros, A.; López-Rodríguez, R.; Estepa, B.; Álvarez, E.; Fernández, R.; Lassaletta, J. M. J. Am. Chem. Soc. 2012, 134, 4573.

[5] Selected examples, see:

(a) Liu, L.; Wang, G.; Jiao, J.; Li, P. Org. Lett. 2017, 19, 6132.

(b) Yang, Y.; Gao, Q.; Xu, S. Adv. Synth. Catal. 2019, 361, 858.

(c) Yamamoto, T.; Ishibashi, A.; Suginome, M. Org. Lett. 2017, 19, 886 .

(d) Crawford, K. M.; Ramseyer, T. R.; Daley, C. J. A.; Clark, T. B. Angew. Chem. Int. Ed. 2014, 53, 7589.

(e) Boebel, T. A.; Hartwig, J. F. J. Am. Chem. Soc. 2008, 130, 7534.

[6] Selected examples, see:

(a) Zeng, J.; Naito, M.; Torigoe, T.; Yamanaka, M.; Kuninobu, Y. Org. Lett. 2020, 22, 3485.

(b) Bai, S.-T.; Bheeter, C. B.; Reek, J. N. H. Angew. Chem. Int. Ed. 
2019, 58, 13039.

(c) Roosen, P. C.; Kallepalli, V. A.; Chattopadhyay, B.; Singleton, D. A.; Maleczka, R. E.; Smith, M. R. J. Am. Chem. Soc. 2012, 134, 11350 .

(d) Preshlock, S. M.; Plattner, D. L.; Maligres, P. E.; Krska, S. W.; Maleczka, R. E.; Smith, M. R. Angew. Chem. Int. Ed. 2013, 52, 12915.

(e) Smith, M. R.; Bisht, R.; Haldar, C.; Pandey, G.; Dannatt, J. E.; Ghaffari, B.; Maleczka, R. E.; Chattopadhyay, B. ACS Catal. 2018, 8,6216 .

(f) Kuninobu, Y.; Ida, H.; Nishi, M.; Kanai, M. Nat. Chem. 2015, 7, 712 .

[7] Chattopadhyay, B.; Dannatt, J. E.; Andujar-De Sanctis, I. L.; Gore, K. A.; Maleczka, R. E.; Singleton, D. A.; Smith, M. R. J. Am. Chem. Soc. 2017, 139, 7864.

[8] (a) Mihai, M. T.; Williams, B. D.; Phipps, R. J. J. Am. Chem. Soc. 2019, 141, 15477 .

(b) Davis, H. J.; Genov, G. R.; Phipps, R. J. Angew. Chem. Int. Ed. 2017, 56, 13351.

(c) Davis, H. J.; Mihai, M. T.; Phipps, R. J. J. Am. Chem. Soc. 2016, $138,12759$.

[9] Yang, L.; Semba, K.; Nakao, Y. Angew. Chem. Int. Ed. 2017, 56, 4853.

[10] (a) Liskey, C. W.; Hartwig, J. F. J. Am. Chem. Soc. 2012, 134, 12422.

(b) Oeschger, R.; Su, B.; Yu, I.; Ehinger, C.; Romero, E.; He, S.; Hartwig, J. Science 2020, 368, 736.

(c) Zhong, R.-L.; Sakaki, S. J. Am. Chem. Soc. 2019, 141, 9854.

(d) Larsen, M. A.; Oeschger, R. J.; Hartwig, J. F. ACS Catal. 2020, 10,3415 .

(e) Ohmura, T.; Torigoe, T.; Suginome, M. J. Am. Chem. Soc. 2012, 134, 17416.

(f) Kawamorita, S.; Murakami, R.; Iwai, T.; Sawamura, M. J. Am. Chem. Soc. 2013, 135, 2947.

[11] Cho, S. H.; Hartwig, J. F. J. Am. Chem. Soc. 2013, 135, 8157.

[12] Kang, E.; Kim, H. T.; Joo, J. M. Org. Biomol. Chem. 2020, 18, 6192.

[13] Boerth, J. A.; Hummel, J. R.; Ellman, J. A. Angew. Chem. Int. Ed. 2016, $55,12650$.

[14] (a) Gulia, N.; Daugulis, O. Angew. Chem. Int. Ed. 2017, 56, 3630. (b) Yang, W.; Ye, S.; Fanning, D.; Coon, T.; Schmidt, Y.; Krenitsky, P.; Stamos, D.; Yu, J.-Q. Angew. Chem. Int. Ed. 2015, 54, 2501. (c) Shabashov, D.; Daugulis, O. Org. Lett. 2005, 7, 3657. (d) Hull, K. L.; Sanford, M. S. J. Am. Chem. Soc. 2007, 129, 11904. (e) Shen, Y.; Cindy Lee, W.-C.; Gutierrez, D. A.; Li, J. J. J. Org. Chem. 2017, 82, 11620.

[15] (a) Ackermann, L.; Althammer, A.; Born, R. Tetrahedron 2008, 64, 6115.

(b) Ackermann, L.; Althammer, A.; Born, R. Angew. Chem. Int. Ed. 2006, 45, 2619.

(c) Hofmann, N.; Ackermann, L. J. Am. Chem. Soc. 2013, 135, 5877 .

(d) Wang, Y.; Liu, H.; Li, B.; Wang, B. Adv. Syn. Catal. 2019, 361, 1564.

[16] (a) Yuan, C.; Tu, G.; Zhao, Y. Org. Lett. 2017, 19, 356

(b) Li, T.; Liu, C.; Wu, S.; Chen, C.; Zhu, B. Org. Biomol. Chem. 2019, 17, 7679.

(c) Cai, H.; Thombal, R. S.; Li, X.; Lee, Y. R. Adv. Synth. Catal. 2019, 361,4022 .

(d) Kim, H.; Thombal, R. S.; Khanal, H. D.; Lee, Y. R. Chem. Commun. 2019, 55, 13402.

(e) Saitou, T.; Jin, Y.; Isobe, K.; Suga, T.; Takaya, J.; Iwasawa, N. Chem. Asian J. 2020, 15, 1941.

[17] Gao, P.; Guo, W.; Xue, J.; Zhao, Y.; Yuan, Y.; Xia, Y.; Shi, Z. J. Am. Chem. Soc. 2015, 137, 12231.

[18] (a) Wang, Y.; Liu, H.; Li, B.; Wang, B. Adv. Synth. Catal. 2019, $361,1564$.

(b) Botla, V.; Akudari, A.; Malapaka, C. Tetrahedron Lett. 2019, 60, 115 .

(c) Lee, W.-C. C.; Shen, Y.; Gutierrez, D. A.; Li, J. J. Org. Lett. 2016, 18, 2660.

[19] Crystallographic data for $\mathbf{1 3}$ could be found in the Supporting Information. CCDC 2031418 (13) contains the supplementary crystallographic data for this paper. The data can be obtained free of charge from The Cambridge Crystallographic Data Center via www. ccdc. cam. ac. uk/data_request/cif.

[20] Hu, N.; Zhao, G.; Zhang, Y.; Liu, X.; Li, G.; Tang, W. J. Am. Chem. Soc. 2015, 137, 6746.

[21] Liu, Z.; Ni, H.-Q.; Zeng, T.; Engle, K. M. J. Am. Chem. Soc. 2018, $140,3223$.

[22] Kawamorita, S.; Miyazaki, T.; Iwai, T.; Ohmiya, H.; Sawamura, M. J. Am. Chem. Soc. 2012, 134, 12924.

[23] Bonet, A.; Odachowski, M.; Leonori, D.; Essafi, S.; Aggarwal, V. K. Nat. Chem. 2014, 6, 584.

[24] Kremsner, J. M.; Kappe, C. O. J. Org. Chem. 2006, 71, 4651.

(Li, L.; Fan, Y.) 